\title{
Identifying the molecular systems that influence cognitive resilience to Alzheimer's disease in genetically diverse mice
}

\author{
Sarah E. Heuer, ${ }^{1,2}$ Sarah M. Neuner, ${ }^{1,3}$ Niran Hadad, ${ }^{1}$ Kristen M.S. O'Connell, ${ }^{1}$ \\ Robert W. Williams, ${ }^{3}$ Vivek M. Philip, ${ }^{1}$ Chris Gaiteri, ${ }^{4}$ and Catherine C. Kaczorowski ${ }^{1,2}$ \\ ${ }^{1}$ The Jackson Laboratory, Bar Harbor, Maine 04609, USA; ${ }^{2}$ Tufts University School of Graduate Biomedical Sciences, Boston, \\ Massachusetts 02111, USA; ${ }^{3}$ University of Tennessee Health Science Center, Memphis, Tennessee 38163, USA; ${ }^{4}$ Rush Alzheimer's \\ Disease Center, Rush University Medical Center, Chicago, Illinois 60612, USA
}

\begin{abstract}
Individual differences in cognitive decline during normal aging and Alzheimer's disease (AD) are common, but the molecular mechanisms underlying these distinct outcomes are not fully understood. We utilized a combination of genetic, molecular, and behavioral data from a mouse population designed to model human variation in cognitive outcomes to search for the molecular mechanisms behind this population-wide variation. Specifically, we used a systems genetics approach to relate gene expression to cognitive outcomes during $A D$ and normal aging. Statistical causal-inference Bayesian modeling was used to model systematic genetic perturbations matched with cognitive data that identified astrocyte and microglia molecular networks as drivers of cognitive resilience to AD. Using genetic mapping, we identified Fgf2 as a potential regulator of the astrocyte network associated with individual differences in short-term memory. We also identified several immune genes as regulators of a microglia network associated with individual differences in long-term memory, which was partly mediated by amyloid burden. Finally, significant overlap between mouse and two different human coexpression networks provided strong evidence of translational relevance for the genetically diverse AD-BXD panel as a model of late-onset AD. Together, this work identified two candidate molecular pathways enriched for microglia and astrocyte genes that serve as causal AD cognitive biomarkers, and provided a greater understanding of processes that modulate individual and population-wide differences in cognitive outcomes during $A D$.
\end{abstract}

[Supplemental material is available for this article.]

Alzheimer's disease (AD) dementia is a common neurodegenerative disorder defined by extracellular amyloid plaques and intracellular neurofibrillary tangles, and characterized by progressive memory decline (Selkoe 1991). Many of the tested therapies for $\mathrm{AD}$ have focused on targeting and clearing pathological beta-amyloid $(\mathrm{A} \beta)$, which has been extensively researched as an initiating factor that leads to neurodegeneration and cognitive impairment (Hardy and Selkoe 2002; Selkoe and Hardy 2016). To date, therapeutics to delay or reverse the progression of AD-related cognitive decline have been disappointing, despite significantly removing $A \beta$ burden (Gao et al. 2016; Elmaleh et al. 2019). These drug failures, and investigations into the origin of $\mathrm{AD}$ more broadly, are both impacted by the divergence between the clinical (cognitive decline) and pathological (amyloidosis and hyperphosphorylated tau) presentation of the disease, with some cognitively intact elderly individuals exhibiting significant amyloidosis consistent with new diagnostic criteria for AD (Morris et al. 1996; Budson and Solomon 2012; Negash et al. 2013). Even individuals with well-defined familial AD (FAD) mutations in amyloid precursor protein $(A P P)$ or presenilin 1 (PSEN1) genes can maintain cognitive function well beyond their sixth decade, despite robust amyloid and tau pathological burden, identifying individuals that are resilient to pathology-induced cognitive decline (Crystal et al. 1988; Katzman et al. 1988; Ryman et al. 2014;

Corresponding author: catherine.kaczorowski@jax.org

Article is online at http://www.learnmem.org/cgi/doi/10.1101//m.051839.120. Freely available online through the Learning \& Memory Open Access option.
Arenaza-Urquijo and Vemuri 2018). In light of this disconnect between $\mathrm{AD}$ pathology and cognitive symptoms, there is a critical need to understand how genetic background can modify the impact of amyloid on variation in $\mathrm{AD}$ cognitive decline.

Recent advances in genomic techniques have enabled detailed investigation of cognitive decline using genome-wide transcriptional profiling from postmortem brains of demented and nondemented control individuals (Miller et al. 2013; Zhang et al. 2013; Narayanan et al. 2014; De Jager et al. 2018). Although these studies have identified some of the processes associated with cognitive decline, such as increased brain inflammation and neurodegenerative pathways, there is still an incomplete understanding of the factors that directly drive cognitive outcomes in dementia patients. One recent study used an integrative approach which combined postmortem gene coexpression, measures of cognitive decline, and measures of pathology to generate a Bayesian conditional-dependency network that identified several modules predicted to directly influence cognitive decline without pathology (Mostafavi et al. 2018). This was a key development in understanding the complex etiology of AD-related cognitive decline in that rather than identifying correlational relations, this study used Bayesian directed modeling that incorporated molecular networks, pathological, and cognitive measures to propose a candidate biological network that directly influenced cognitive decline

(C) 2020 Heuer et al. This article, published in Learning \& Memory, is available under a Creative Commons License (Attribution-NonCommercial 4.0 International), as described at http://creativecommons.org/licenses/by-nc/4.0/. 
without the direct influence of pathology. However, the reliance on postmortem tissues limits our ability to define early molecular changes that may drive onset of dementia, especially since many of these processes start long before the onset of cognitive symptoms (Fox et al. 1996; Paterson et al. 2014; Hohman et al. 2017).

To identify novel genetic factors and molecular systems that are responsible for resilience to cognitive symptoms of AD longitudinally and under controlled environmental conditions, we systematically examined the effect of FAD mutations on cognitive traits in a genetically diverse panel of mice; the AD-BXDs (Neuner et al. 2019a). The AD-BXD genetically diverse mouse model of AD incorporates the commonly used 5XFAD transgene across a number of diverse, yet inbred, mouse strains from the BXD genetic reference panel (Neuner et al. 2019a). We found that the AD-BXDs exhibited variation in cognitive decline similar to that observed in human FAD populations (Ryman et al. 2014). This variation was sensitive to genetic risk factors associated with human sporadic AD (Neuner et al. 2019a), suggesting this is a translationally relevant model of late-onset AD (LOAD).

The goal of this study is to build on our previous findings to further prioritize the molecular systems which likely account for the wide range of cognitive outcomes using a population of identical individuals with and without $\mathrm{AD}$ mutations. We took a systems biology approach combining genetic, transcriptomic, and behavioral data from a large population of normal aging (Ntg-BXD) and $\mathrm{AD}$ (AD-BXD) cases (Fig. 1). Specifically, we inferred gene coexpression modules from hippocampus transcriptome data of a genetically diverse mouse population from young [6 mo old $(6 \mathrm{mo})]$ and aged [14 mo old (14 mo)] individuals. Each of these modules represents the activity of one or more molecular systems, and was identified in a purely data-driven manner, blind to genotype or behavioral outcomes. Then, we identified which modules and molecular systems were associated with age, amyloid mutations, and cognitive outcomes. Genetic mapping of module eigengene expression as quantitative traits identified genetic regions that regulated each module. We then used Bayesian conditional dependency modeling (Tasaki et al. 2015) to eliminate redundant associations and predict the subset of modules driving individual differences in cognition across the population, and calculated if these relations were dependent on the presence of amyloid-driving mutations, and if module-trait relations were strengthened by the addition of cortical amyloid burden into our statistical model. Finally, we tested if the prioritized molecular networks from this study are translationally relevant by testing the overlap between mouse and human dementia networks (Bennett et al. 2012; Mostafavi et al. 2018; Wan et al. 2020). This approach nominates robust molecular systems that consistently relate to individual differenc- es in cognition in an aging and $\mathrm{AD}$ populations, and evaluates the effect of these systems on cognitive outcomes. Furthermore, additional modeling that incorporated cortical A $\beta 1-42$ ELISA suggests an indirect relation of $\mathrm{AD}$ pathological burden on cognitive outcomes in promoting differences in molecular network activity, and highlights the utility of mouse models for discovering several molecular systems to monitor for resilience and manipulate them in future validation studies to improve $\mathrm{AD}$ cognitive outcomes.

\section{Results}

Defined molecular systems of the hippocampus in AD-BXD and Ntg-BXD mice

In order to identify the molecular systems associated with cognitive outcomes across the population of both $\mathrm{AD}$ and non-AD mouse strains, we first obtained RNA sequencing (RNA-seq) data
A
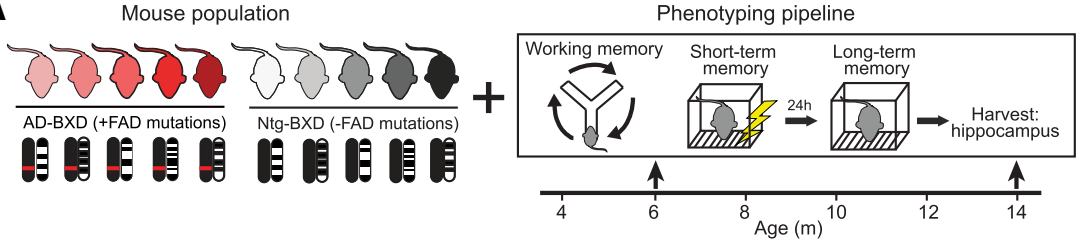

B
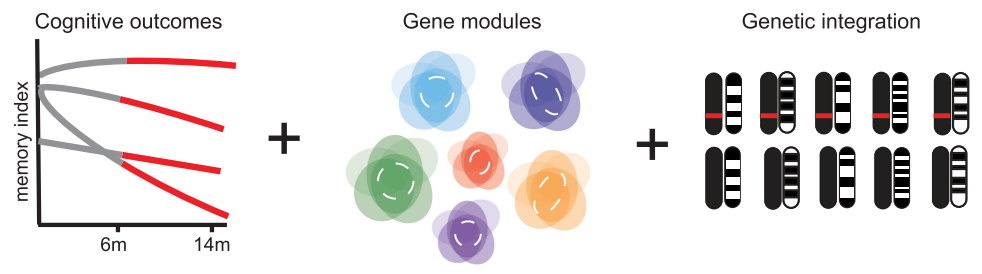

C

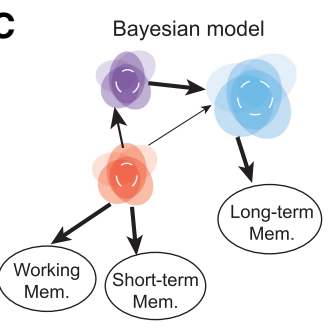

D

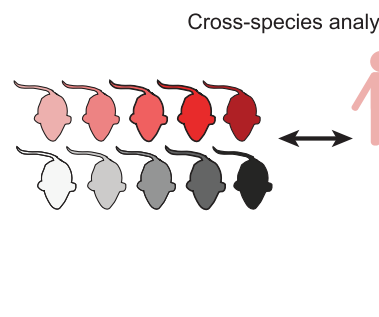

Figure 1. Genetically diverse mice can be leveraged to understand the relevant molecular networks that underlie individual differences in cognitive outcomes. Schematic of SpeakEasy module formation and relation to cognitive outcomes, adapted from Mostafavi et al. (2018). (A) Multiple strains of genetically diverse mice that are carriers (AD-BXD) or noncarriers (Ntg-BXDs) for the 5XFAD transgene (FAD mutations) are combined to generate a population with genetic diversity similar to what is observed in humans. These strains make up a genetic reference panel, allowing for the incorporation of genetic information into further analyses. Each mouse is brought through a cognitive phenotyping pipeline (described in Neuner et al. 2019a), taking measures for working memory, short-term memory, and long-term memory at 6 mo or 14 mo of age, and harvest of hippocampal tissue for downstream analyses. $(B)$ Measures of the three cognitive domains taken during the phenotyping pipeline are assessed from AD-BXD and Ntg-BXD mice at 6 mo and 14 mo of age (left), while gene coexpression modules are separately formed from the same mice using SpeakEasy consensus clustering (middle). Additionally, genetic information is obtained from each strain for downstream genetic mapping and integration into the module-trait network (right). (C) Gene expression modules are related to cognitive traits using Spearman correlations, and modules significantly (adj. $P<0.05$ ) correlated to at least one cognitive measure are selected for downstream analysis. These cognitively relevant modules are integrated with cognitive measures, and anchored by BXD genetics to form a module-trait Bayesian directed network, and modules directly upstream of cognitive traits are prioritized for further characterization. (D) Cognitively relevant modules are compared with human brain modules to investigate gene-gene conservation between mouse and human. 
from the hippocampus of AD-BXD and Ntg-BXD mice at either 6 or 14 mo of age $(n=294)$ (Neuner et al. 2019a,b). Biological replicates from the strain, sex, and genotype groups were averaged together, generating a total of 156 unique group-averaged measures. With these data, we then performed unsupervised consensus clustering and identified 28 discrete transcriptional modules of $>29$ genes, ranging in membership size from 42 to 1419 transcripts (Supplemental Table S1). We commonly refer to these modules as gene sets, but recognize that in this context we more formally mean sets of mRNAs with one-to-one relations to genes. We chose the SpeakEasy consensus clustering algorithm over other clustering algorithms, such as weighted gene coexpression network analysis (WGCNA), because it is less parameter-driven and carries a higher degree of run-to-run stability for transcriptome network conservation (Gaiteri et al. 2015; Mostafavi et al. 2018). To gain a basic understanding of the biological processes and cell-types enriched in each module, we performed gene ontology (GO) biological process enrichment on each module (Supplemental Table S2), as well as cell-type enrichment using a well-established and mineable RNA-seq data set from mouse cortex (Zhang et al. 2014). The major molecular functions and module-module relations of these gene sets are highlighted in Figure 2. Modules that were clustered together based on covariation in expression are also similarly char- acterized based on cell-type and biological function, without having overlapping members since each module was formed using discrete gene assignment. These included a group of modules enriched for genes expressed in neurons, as well as synaptic organization and intracellular transport GO terms, a large grouping of astrocyte modules enriched for RNA processing, cell movement, and chromatin remodeling GO terms, a mixed endothelial cell and astrocyte grouping of modules enriched for metabolism GO terms, and a microglia module group enriched for immune function GO terms (Fig. 2). The annotation of these covarying molecular systems identifies the major cell-types and biological pathways present in the hippocampus of our population, a subset of which may change as a function of aging and/or disease processes.

\section{Prioritizing molecular systems related to cognitive outcomes highlights immune and metabolic networks}

The coexpression analysis described above defined the basic molecular systems that can be robustly identified and evaluated in mouse hippocampi. Importantly, these mRNA sets are formed without any reference to genotype or phenotype. Therefore, we next considered how the average expression of each coexpression module was related to sex, age, and FAD mutation carrier status

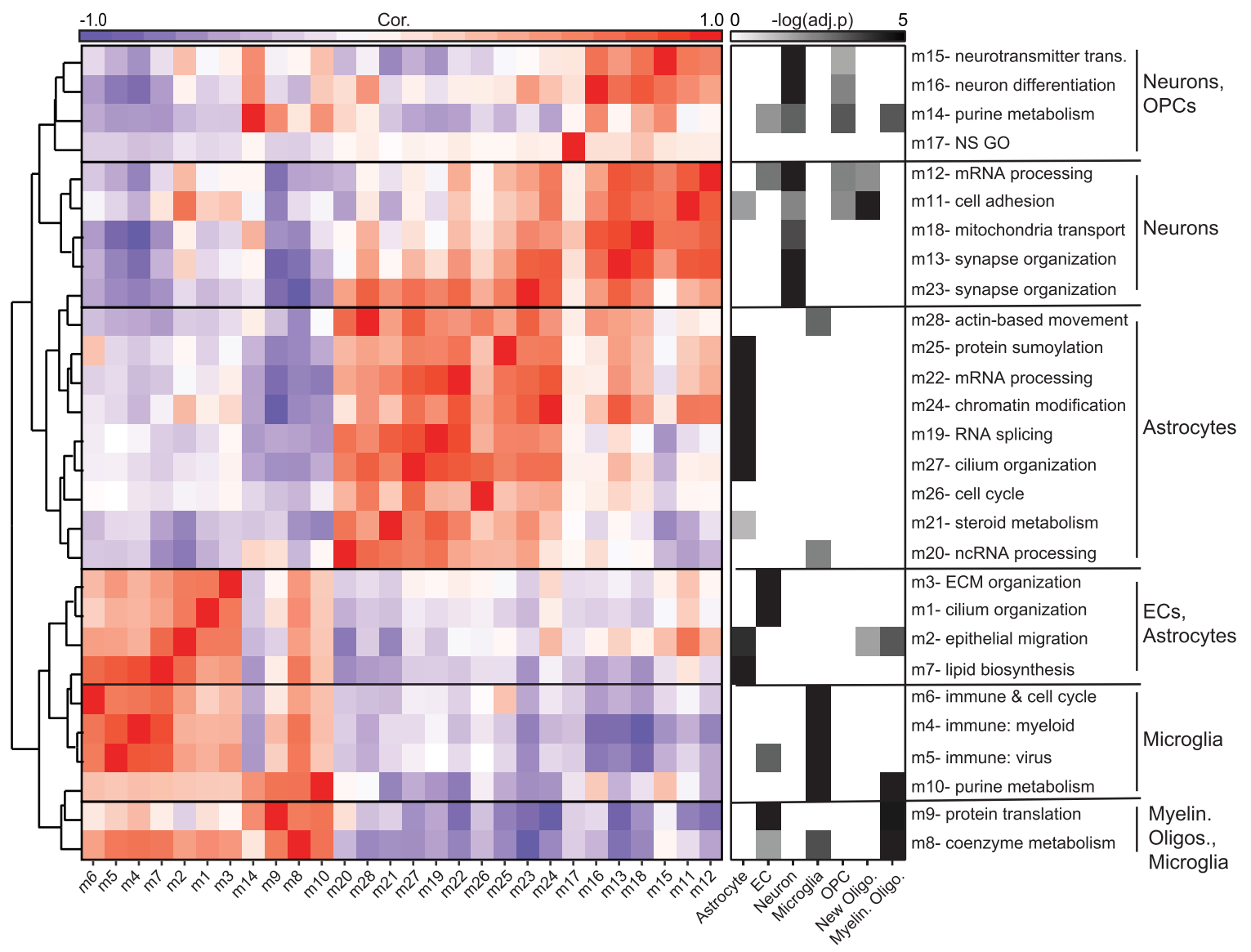

Figure 2. $A D-B X D$ and Ntg-BXD have variable transcriptional networks across aging and Alzheimer's individuals. SpeakEasy consensus clustering identified 31 discrete transcriptional modules from hippocampal RNA sequencing of Ntg/AD-BXD mice ( $n=26$ strains). Modules containing $<30$ genes were excluded ( $n=3$ modules). Average module expression per sample was derived using average standardized gene expression of module members. Modules were correlated to each other (Pearson) and hierarchically clustered. Cell-type enrichment was performed on the genes within each module using the Zhang et al. (2014) data set to obtain an idea of represented cell-types for each module. Cell-type enrichments were performed using hypergeometric tests to identify significant relationships (adj. $P<0.05)$ for astrocytes, endothelial cells $(E C)$, neurons, microglia, oligodendrocyte precursor cells $(O P C)$, new oligodendrocytes (New Oligo.), and myelinating oligodendrocytes (Myelin. Oligo.). GO enrichment was performed on the genes in each module, and the GO term most significantly enriched within a module is denoted (NS GO = no significant $\mathrm{GO}$ enrichment). 
(Supplemental Figs. S1-S3), and cognitive status (Fig. 2). To do this, we first generated a summary statistic for each module by calculating the average standardized expression of the gene members for each module. Next, we compared each module summary statistic with strain-averaged performance on cognitive tasks using Spearman correlation analysis. These tasks spanned multiple cognitive domains and included working memory using spontaneous alternation on the Y-maze and acquisition and recall of contextual fear conditioning at both 6 and 14 mo of age. Spontaneous alternation relies on a rodent's innate tendency to explore novel environments while avoiding familiar environments and assesses hippocampal- and cortical-dependent spatial working memory. This task was initially chosen due to the lack of need for training, reward, or punishment, and the ability to test mice on this task longitudinally (Holcomb et al. 1998; Ohno et al. 2004; Oakley et al. 2006). Contextual fear conditioning enables the assessment of both hippocampal-dependent short-term memory during the acquisition phase in which mice are administered four mild foot shocks (Chen et al. 1996; Neuner et al. 2015), as well as hippocampal-dependent spatial orientation, recall, and long-term memory during the testing phase in which mice are placed back into the training chamber in the absence of foot shocks $24 \mathrm{~h}$ after contextual fear conditioning training (Kim and Jung 2006; Neuner et al. 2015).

We identified 16 modules that significantly correlated with at least one cognitive outcome (Fig. 3A; Spearman, adj. $P<0.05$ ).

A

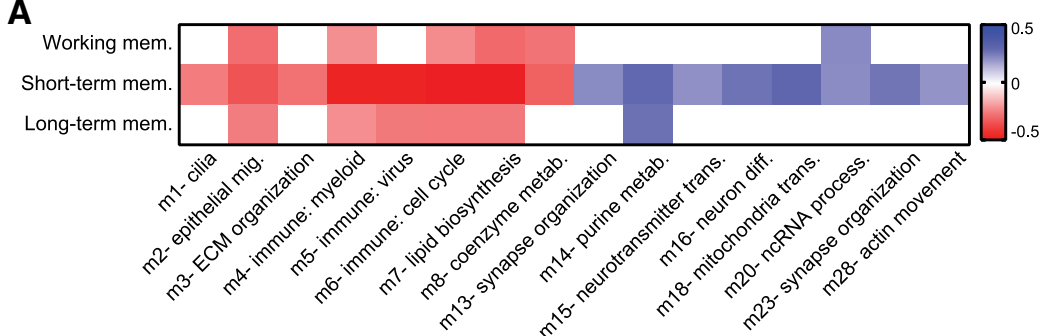

B

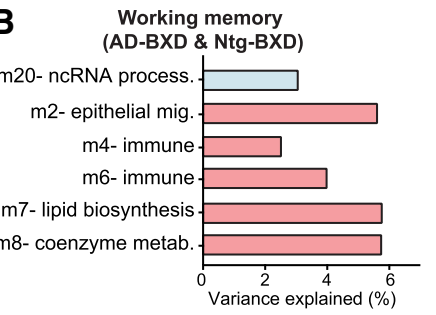

C

Short-term memory
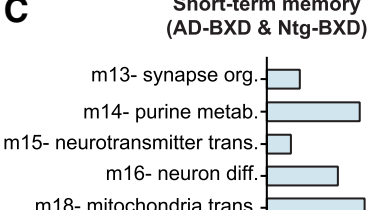

m20-ncRNA process

m23- synapse org.

m28- actin movement-

$$
\text { m1- cilia- }
$$

$\mathrm{m} 2$ - epithelial mig.

m3- ECM org.

m4-immune-

m5- immune-

m6-immune

m7- lipid biosynthesis

m8- coenzyme metab.

m7- lipid biosynthesis

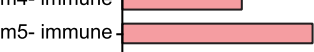

m6-immune

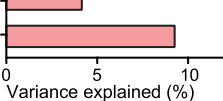

There were 6 modules ( 5 negative, 1 positive) significantly correlated with percent successful spontaneous alternations (\%SA) on the Y-maze working memory task. The modules negatively correlated with \%SA on the Y-maze working memory task $(\mathrm{m} 2, \mathrm{~m} 4, \mathrm{~m} 6$, $\mathrm{m} 7, \mathrm{~m} 8)$ contain genes highly expressed in microglia, and enriched for GO biological processes including cell migration, immune function, and lipid biosynthesis processes. The only module positively correlated with \%SA on Y-maze (m20) contained members that are also expressed in microglia and were enriched for noncoding RNA processing GO terms. There were 16 modules ( 8 negative, 8 positive) that significantly correlated with contextual fear acquisition (CFA) slope (see Materials and Methods). The negatively correlated modules contained genes expressed in astrocytes and microglia and were primarily enriched for GO terms associated with immune function $(\mathrm{m} 4, \mathrm{~m} 5, \mathrm{~m} 6)$ as well as cell migration ( $\mathrm{m} 1, \mathrm{~m} 2, \mathrm{~m} 3)$ and lipid biosynthesis and metabolism (m7, m8). The positively correlated modules contained genes expressed primarily in neurons and were enriched for GO processes associated with synaptic signaling and organization (m13, m15, $\mathrm{m} 16, \mathrm{~m} 23, \mathrm{~m} 28)$, mitochondrial transport (m18), and RNA processing and metabolism (m14, m20). Finally, there were 6 modules ( 5 negative, 1 positive) significantly correlated with contextual fear memory (CFM). The negatively correlated modules contained genes expressed in microglia and astrocytes and were enriched for migration ( $\mathrm{m} 2)$, immune ( $\mathrm{m} 4, \mathrm{~m} 5, \mathrm{~m} 6)$ and lipid biosynthesis $(\mathrm{m} 7)$ processes. The positively correlated module (m14) was enriched for genes associated with purine metabolism processes and contained genes expressed in multiple cell-types including endothelial cells, neurons, microglia, and myelinating oligodendrocytes. One thing to note is that we identified four modules that were significantly correlated with all three cognitive outcomes $(\mathrm{m} 2, \mathrm{~m} 4, \mathrm{~m} 6, \mathrm{~m} 7)$, suggesting perturbation of these modules would influence all three cognitive domains, instead of being-domain specific processes. Together, results from these analyses suggest that increased number of transcripts associated with antiviral and innate immune pathways in microglia, as well as lipid biosynthesis pathways in astrocytes associate with lower cognitive performance, while synaptic signaling and purine metabolism pathways that are enriched in neuron and oligodendrocyte precursor cells are associated with higher cognitive performance, highlighting these biological networks as resilience candidates.

In addition to identifying significant module-trait relations, we also tested the extent to which significantly correlated modules explained variance in cognitive outcomes across the population. Initially, we calculated heritability of each trait to determine the amount of variance genetic background (or BXD strain) explained for each cognitive outcome. Similar to our previous findings (Neuner et al. 2019a), the majority of the variance in cognitive outcomes could be explained by genetic background, with $73 \%$ heritability for \%SA on Y-maze working memory, 70\% heritability for CFA slope, and $89 \%$ heritability for CFM. Using three- 
way ANOVA analyses we separately calculated the amount of variance in each cognitive outcome that could be explained by binarycoded variables such as age, sex and presence of FAD mutations (Supplemental Table S3). Age was the only variable that significantly affected \%SA on Y-maze working memory, explaining $8.2 \%$ of the variance in \%SA. Additionally, age was the only variable explaining significant variance for CFM, explaining $12.7 \%$ of the variance in \%freezing on this task. Finally, age and FAD mutation carrier status explained significant variance for CFA slope, with $4.6 \%$ explained by age, and $19.9 \%$ explained by FAD mutation carrier status.

Using linear regression modeling, the variance of each cognitive outcome explained by module expression was calculated and used as a proxy for effect size of module expression on cognitive outcomes. We focused only on module-trait relationships that reached statistical significance in our Spearman correlation analysis, and performed individual linear regressions for each moduletrait pair. The modules that explained the most variance for working memory (\%SA) outcomes were the epithelial migration module (m2, 5.6\%), lipid biosynthesis module (m7, 5.8\%) and coenzyme metabolism module (m8, 5.7\%) (Fig. 3B). Variance in CFA shortterm memory outcomes was explained more strongly and by a larger number of modules than \%SA, including immune modules ( $\mathrm{m} 4$, $24.0 \% ; \mathrm{m} 5,16.0 \%, \mathrm{~m} 6,21.3 \%)$, and the lipid biosynthesis module (m7, 27.8\%) (Fig. 3C). The modules that explained the greatest amount of variance for CFM long-term memory outcomes included the immune: virus module (m5, 10.5\%), lipid biosynthesis module (m7, 9.3\%), and purine metabolism module (m14, 7.7\%) (Fig. $3 \mathrm{D})$. While these module-trait correlations can prioritize molecular systems in disease, the causal nature of these relationships is unclear. Therefore, we proceeded to integrate genetic priors and conditional dependences to further assess the robustness of the top findings from these initial results.

\section{Genetic network analysis supports a role for lipid biosynthesis and viral immune enriched molecular networks in cognitive resilience}

As the effect of a molecular system on a phenotype may be conditional on activation of other molecular systems, we tested for such relations in the module-trait associations we identified. In this analysis, we simultaneously utilized module expression for the 16 cognitive relevant modules, cognitive measures, as well as information about genomic regions involved in regulating module expression. Chromosomal regions that regulate module expression were identified using genetic mapping in which module eigengenes were used as quantitative traits. According to the central dogma of expression genetics (Crick 1958; Williams et al. 2007), DNA variants influence RNA expression, which then modulates downstream behavioral phenotypes such as cognitive outcomes (Hsu et al. 2007). As such these chromosomal regions were used as causal anchor points in our Bayesian network analysis to attempt to identify which molecular networks (and chromosomal regions) were causally associated with cognitive outcomes. The modules predicted to directly influence cognitive outcomes were $\mathrm{m} 7$ (astrocytes, lipid biosynthesis) for Y-maze working memory and CFA short-term memory and m5 (microglia, immune: virus) for CFM long-term memory (Fig. 4).

The module directly upstream of Y-maze working memory and CFA short-term memory, $\mathrm{m} 7$, also explained the most variance in these cognitive measures (Fig. 3B,C). Module m7 contained 243 gene members, making it smaller in size in comparison to the three major microglia modules with 1088 members in m4, 848 members in $\mathrm{m} 5$, and 681 members in m6. This module was most significantly enriched for GO terms related to lipid biosynthesis and for genes that are highly expressed in astrocytes (Fig. 2). Ingenuity

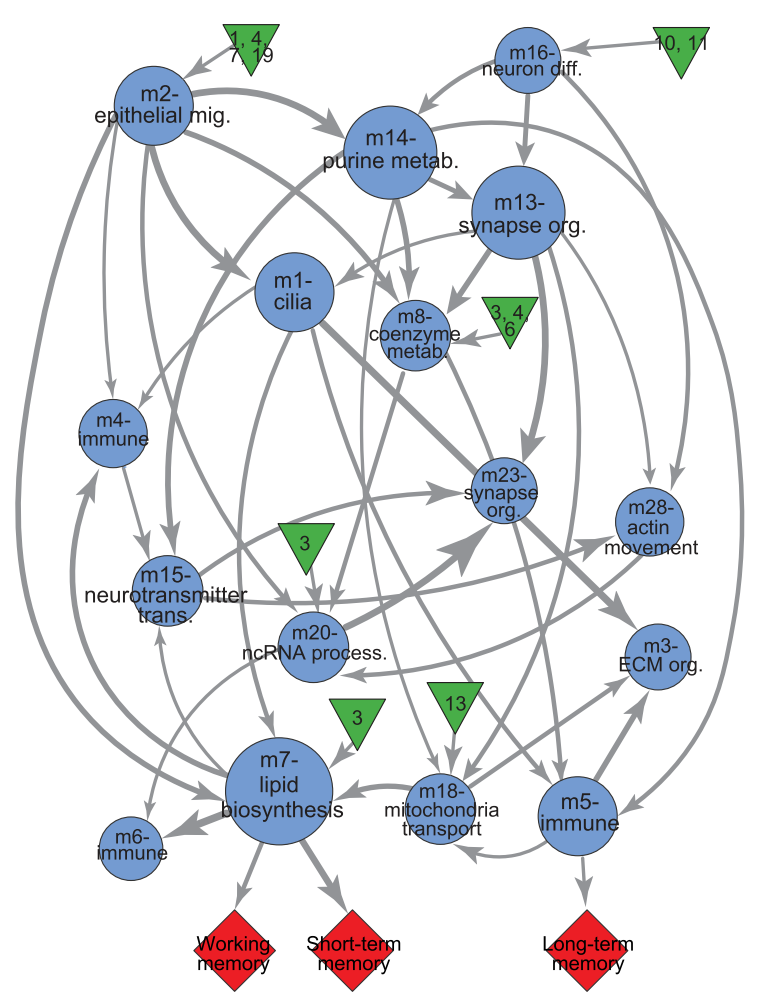

Figure 4. Bayesian conditional dependency modeling of cognitively relevant modules and traits defines causal module-trait relationships. Genetics (green triangles), module expression (blue circles, denoted by most significantly enriched GO term), and measures for each cognitive domain (red diamonds) were combined into a single module-trait network using Bayesian conditional dependency modeling. The genomic priors used in this analysis were the identified genetic regions associated with module eigengenes (PC1), and the chromosome(s) that each region mapped to is denoted inside each triangle. The thickness of the arrows represents the number of times a module-module or module-trait relationship was detected.

pathway analysis (IPA) identified multiple molecular systems enriched in this gene set consistent with astrocyte activity including Interleukin-1 (IL-1) signaling, cholesterol biosynthesis and actin cytoskeleton signaling (Table 1). Specifically, the $\mathrm{m} 7$ genes enriched in the IL-1 signaling pathway were Irak2 and Irak3, part of the IRAK family of proteins that are involved in direct downstream signaling of the IL-1 receptor, as well as Rhoa, which is important to actin cytoskeleton signaling.

The module directly upstream of CFM long-term memory outcomes was m5, enriched for GO biological processes associated with viral immune response (Fig. 2). This module consisted of 848 gene members, making it one of the largest modules constructed in this analysis. Enrichment of this module for GO biological processes and IPA canonical pathways highlighted the primary function of this module was proinflammatory immune signaling. Specifically, many of the enriched IPA canonical pathways referenced pathways involved in immune responses to virus, such as "role of pattern recognition receptors in recognition of bacteria and viruses," "interferon signaling," and "acute phase response signaling" (Table 2), confirming the primary function of this module as immune response. Upon further inspection of module members that also participated in these pathways, many are members of the interferon response pathway (e.g., Oas1, Oas1b, Oas2, Oas3, Eif2ak2, and Ifih1) which is the primary pathway activated upon viral infection (Kawai and Akira 2006, 2007). 
Table 1. Top Five IPA enriched canonical pathways for module 7 ( $\mathrm{m7}$, lipid biosynthesis)

\begin{tabular}{|c|c|c|}
\hline IPA canonical pathway & $P$-value & Genes \\
\hline $\begin{array}{l}\text { Role of macrophages, fibroblasts and endothelial cells in } \\
\text { rheumatoid arthritis }\end{array}$ & $3.89 \times 10^{-6}$ & $\begin{array}{l}\text { Cebpg, Fgf2, Fzd9, II1 7ra, II33, Irak2, Irak3, Nfkbie, Plce1, Rap1b, Rhoa, } \\
\text { Ripk1, Stat3, Tcf7l1 }\end{array}$ \\
\hline Superpathway of cholesterol biosynthesis & $8.13 \times 10^{-6}$ & Dhcr24, Dhcr7, Hmgcs1, Hsd17b7, Idi1 \\
\hline Interleukin-1 (IL-1) signaling & $3.89 \times 10^{-5}$ & Gna12, Gna13, Gng12, Irak2, Irak3, Nfkb2, Nfkbie \\
\hline Actin cytoskeleton signaling & $8.32 \times 10^{-5}$ & Cyfip1, Fgd3, Fgf2, Gna12, Gna13, Gng12, Gsn, Rap1b, Rhoa, Wasf2 \\
\hline Regulation of the epithelial-mesenchymal transition pathway & $1.58 \times 10^{-4}$ & Fgf2, Fgfrl1, Fzd9, Hgf, Nfkb2, Rap1b, Rhoa, Stat3, Tcf7l1 \\
\hline
\end{tabular}

\section{Genetic mapping of $\mathrm{m} 7$ module eigengene identifies Fgf2 as a regulator of $\mathrm{m} 7$ and short-term memory outcomes}

A major advantage of using genetic reference panels to model polygenetic disorders is the ability to perform genetic mapping of complex traits for such disorders, including measures of module expression, to identify chromosomal regions and genes within them that regulate these traits. To identify chromosomal regions regulating module expression we performed quantitative trait loci (QTL) mapping of module eigengenes for all cognitive relevant modules (Fig. 4; Supplemental Table S4, depicted as green triangles). Specifically, we focused on identifying chromosomal regions regulating $\mathrm{m} 5$ and $\mathrm{m} 7$ expression, since these modules were identified through Bayesian modeling as being directly upstream of cognitive outcomes. While we did not identify any QTLs for the $\mathrm{m} 5$ eigengene, we identified a significant QTL for the $\mathrm{m} 7$ eigengene, which fell in a small region on chromosome 3 (36.5-53.3 $\mathrm{Mb}$ ), which means genetic variation within this region corresponds to variation in $\mathrm{m} 7$ expression (Fig. 5A). Within this region of chromosome 3 we identified 45 protein coding genes, only one of which was a member of m7, Fibroblast growth Factor 2 (Fgf2), identifying this gene as a possible regulator of $\mathrm{m} 7$ expression and activity. Investigation of the variants in $F g / 2$ between C57BL/6J and DBA2/J in the Sanger Mouse Genomes database highlighted many single nucleotide polymorphisms (SNPs) between the two strains, the most notable being a possible splice donor and/or missense variant (Keane et al. 2011). Additionally, our data show that hippocampal Fgf2 expression is significantly higher in strains that carry the B allele compared to strains that carry the D allele at the marker SNP closest to the $F g f 2$ gene body (Fig. 5B). Finally, we found that $\mathrm{Fg} / 2$ expression significantly negatively correlated with Y-maze working memory outcomes in strains that carried the B allele at $F g f 2$, but carried no significant relationship with mice that carried the D allele at Fgf2 (Fig. 5C). Comparatively, $\mathrm{Fg} 22$ expression in all strains significantly negatively correlated with CFA short-term memory outcomes (Fig. 5D). Together, this genetic mapping nominated a candidate by which $\mathrm{m} 7$ expression and possibly activity is controlled, and further experiments will determine the exact role by which Fgf2 may control memory outcomes during aging and $\mathrm{AD}$.

\section{Molecular subnetworks for $\mathrm{m} 5$ implicate specific immune pathways and genes as modulators of long-term memory outcomes}

Since a large number of genes ( 848 genes) fell into $\mathrm{m} 5$, the module directly upstream of CFM long-term memory outcomes in the Bayesian network, we conducted a subclustering analyses to resolve smaller molecular networks that may exist within the larger module. Specifically, we performed SpeakEasy clustering analysis on gene expression measures from $\mathrm{m} 5$ members, and identified five distinct subclusters (SCs) within m5 (Fig. 6A; Supplemental Table S5). GO enrichment of the genes within each SC revealed immunological pathways as the top enriched GO term, such as response to virus (SC1), lymphocyte differentiation (SC2), acute inflammation (SC3), Interleukin-8 (IL-8) secretion (SC4), and acrosome reaction (SC5) (all enriched GO terms in Supplemental Table S6). Spearman correlation of each SC to CFA slope short-term memory and CFM long-term memory outcomes highlighted significant negative module-trait correlations for each SC except for SC5, which was only correlated with long-term memory outcomes (Fig. 6B). Furthermore, SC1: response to virus, SC3: acute inflammation and SC4: IL-8 secretion explained comparable variance in CFA slope short-term memory outcomes to m5 expression (Fig. 6C), while SC3 and SC4 explained comparable variance in longterm memory outcomes to m5 expression (Fig. 6D). Finally, while the m5 eigengene alone did not map to a QTL, we identified six QTLs for the SC5 eigengene (Fig. 6E; Supplemental Table S4). The $\mathrm{m} 5$ gene members within these regions were enriched for immune functions, suggesting regions of the genome that contain genes associated with immune function could also be important for regulating how m5 controls long-term memory outcomes, since SC5 was also correlated with long-term memory. Furthermore, when we investigated how m5 SCs were genetically controlled in the AD-BXD population, we found that the SC5 eigengene mapped to a smaller region on chromosome 1 that overlapped with the QTL we identified in Figure 6E (Supplemental Fig. S5C; Supplemental Table S4). This region of chromosome 1 was also enriched for $\mathrm{m} 5$ members associated with the interferon response pathway (Ifi204, Ifi202b), adaptive immune response (Exo1, Fcrl6) and glia signaling (Kcnj10) (Supplemental Fig. S5D). Together, this subclustering analysis further elucidates the overall

Table 2. Top five IPA enriched canonical pathways for module 5 ( $\mathrm{m} 5$, immune: virus)

\begin{tabular}{|c|c|c|}
\hline IPA canonical pathway & $P$-value & Genes \\
\hline $\begin{array}{l}\text { Role of pattern recognition receptors in } \\
\text { recognition of bacteria and viruses }\end{array}$ & $3.98 \times 10^{-12}$ & $\begin{array}{l}\text { C3, Cc15, Cntf, Ddx58, Eif2ak2, Faslg, Ifih1, II1 8, II1 a, II21, Irf7, Ltb, Myd88, Nod2, } \\
\text { Oas1, Oas1b, Oas2, Oas3, Ptx3, TIr3, TIr6, Tnf, Tnfsf10, Tnfsf12 }\end{array}$ \\
\hline $\begin{array}{l}\text { Systemic lupus erythematosus in B cell } \\
\text { signaling pathway }\end{array}$ & $1.00 \times 10^{-10}$ & $\begin{array}{l}\text { Cd72, Cd79a, Cd79b, Cntf, Faslg, Ifih1, Ifit2, Ifit3, Ighm, II18, II1 a, II21, Irf7, Irf9, Isg15, } \\
\text { Isg20, Lilrb3, Ltb, Myc, Myd88, Pik3ap1, Rras, Shc1, Stat1, Stat2, TIr3, Tnf, Tnfsf10, } \\
\text { Tnfsf1 2, Traf1 }\end{array}$ \\
\hline Interferon (IFN) signaling & $1.62 \times 10^{-9}$ & Ifi35, Ifit3, Ifitm3, Irf1, IIf9, Isg15, Oas1, Socs1, Stat1, Stat2, Tap1 \\
\hline Th1 and Th2 activation pathway & $8.32 \times 10^{-9}$ & $\begin{array}{l}\text { Acvr1c, Aph1 a, Cd274, Cd28, Cd3d, Cd3e, Cd3g, Cd8a, Cxcr6, Icam1, Icos, I118, II2rb, } \\
\text { Irf1, Jak3, KIrd1, Socs1, Socs3, Stat1, Stat4, Stat6 }\end{array}$ \\
\hline Acute phase response signaling & $1.70 \times 10^{-8}$ & $\begin{array}{l}\text { A2m, C1r, C1s, C3, Hpx, I11 8, I11a, II1 rn, Itih3, Map2k3, Myd88, Rras, Serpina3, } \\
\text { Serpinf1, Serpinf2, Serping1, Shc1, Socs1, Socs3, Tnf, Tnfrsf1 1b }\end{array}$ \\
\hline
\end{tabular}


A
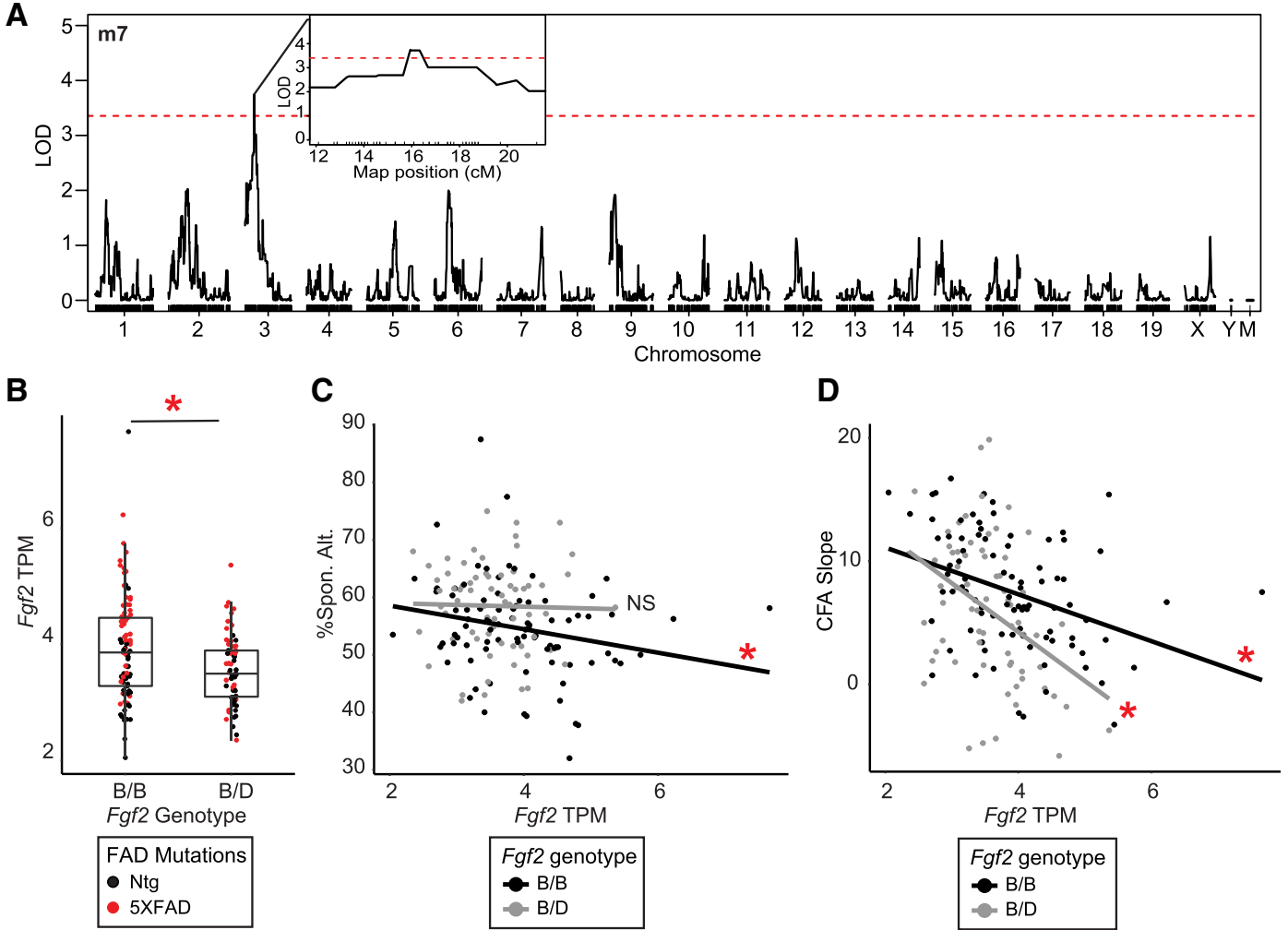

Figure 5. Mapping of the module upstream of working memory and short-term memory measures identifies Fgf2 as a potential driver. (A) Genetic mapping of $\mathrm{m} 7$ using the first principal component (PC1) as module eigengene. Age, sex, and FAD mutation status were used as additive covariates. (B) Stratification of Fgf2 expression based on genotype at the closest marker single nucleotide polymorphism (SNP) to Fgf2 genetic location (Student's $t$-test, $P<0.05$ ). (C) Fgf2 expression is significantly correlated (Spearman, $P<0.05$ ) with overall working memory performance only in mice with the $B / B$ genotype at the marker SNP closest to Fgf2 but not with B/D. (D) Fgf2 expression is significantly correlated (Spearman, $P<0.05$ ) with overall short-term memory performance in mice with either the B/B or and B/D genotype at the marker SNP closest to Fgf2. Significant correlations denoted by red asterisks. NS = no significant correlation.

function of $\mathrm{m} 5$ for viral and adaptive immune response, and nominates a region on chromosome 1 that could be important for controlling m5 expression. Future experiments will deduce the function of this genetic region, and how genes within may control $\mathrm{m} 5$ expression and possibly long-term memory outcomes.

\section{The effect of module expression on AD-BXD long-term memory outcomes is partially modulated by amyloid accumulation}

While the previous analyses were performed on the combined AD-BXD and Ntg-BXD population that contained individual strains that did not carry amyloid-driving mutations, many of the networks explaining large variance in cognition, such as inflammation ( $\mathrm{m} 4$ and $\mathrm{m} 5$ ), related to pathways previously implicated in the etiology of AD. To evaluate how the presence of the 5XFAD transgene affected module expression, we used three-way ANOVA tests on module expression, and found that 11 of the 16 cognitively relevant modules were significantly affected (adj. $P<$ 0.05 ) by presence of the 5XFAD transgene (Supplemental Fig. S1), suggesting that the presence of amyloid-driving mutations accounts for the majority of the significant module--trait relationships. To test if module-trait relationships were primarily driven by the presence of FAD mutations, we recalculated the moduletrait Spearman correlations of the 16 cognitively relevant modules for the Ntg-BXD and AD-BXD populations separately. While there were no significant module-trait correlations in the Ntg-BXD population, we found that 7 of the 16 original cognitively relevant modules continued to be correlated to cognitive outcomes in the AD-BXD population, including $\mathrm{m} 5$ (immune: virus) and $\mathrm{m} 7$ (lipid biosynthesis), which were the modules directly upstream of cognitive outcomes in the Bayesian model (Fig. 7A; Supplemental Table S7). This supported the hypothesis that the presence of amyloiddriving mutations strengthens the module--trait relationships that explain a portion of the individual differences in cognitive outcomes.

To determine the degree to which the seven significant module-trait relationships within the $\mathrm{AD}-\mathrm{BXD}$ population were dependent on the amount of brain amyloid (Fig. 7A), we quantified the amount of variance that cortical amyloid load explained for each cognitive measure within the AD-BXD population (A $31-42$ ELISA assay previously reported in (Neuner et al. 2019a)). Cortical A $31-42$ (ng/mg total protein) explained $3.5 \%$ of working memory (not statistically significant), $9.0 \%$ of short-term memory $(P<$ $0.05)$, and $14.3 \%$ of long-term memory outcomes $(P<0.01)$ (Fig. $7 \mathrm{~B})$. For several modules A $\beta 1-42$ could explain a large amount of the variance in module expression, such as $29.4 \%$ of the variance in $\mathrm{m} 4$ expression, and $26.3 \%$ of the variance in $\mathrm{m} 7$ expression $(P$ $<0.01$ ) (Fig. 7C). This suggested that A $\beta 1-42$ likely influences the expression of modules within AD-BXDs.

To test the degree to which cortical A $\beta 1-42$ modulated the effect of each module (as measured by average expression) had on each cognitive domain, we generated linear models for each significant module-trait relationship, with the addition of cortical A 1 1-42 ELISA measures as a second independent variable within the model. We first used Type 3 ANOVA statistical tests to obtain 
A
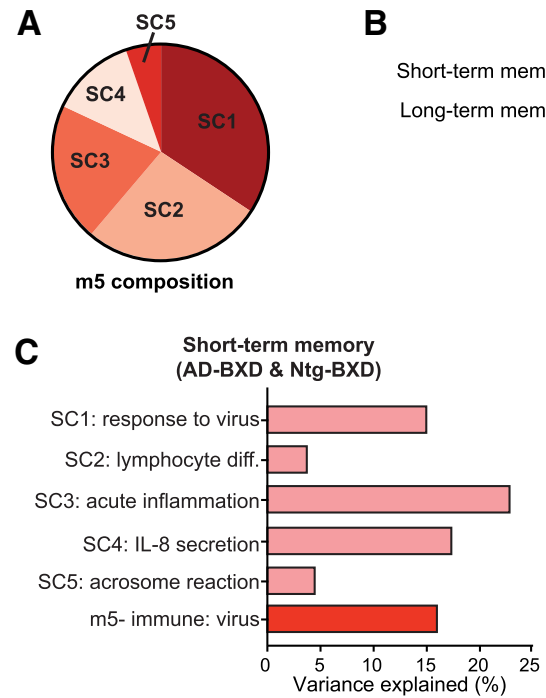

E

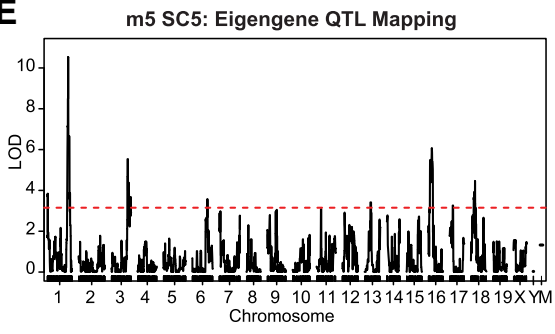

B

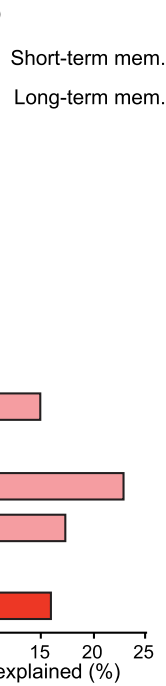

$F$

\begin{tabular}{|c|c|c|}
\hline GeneSymbol & EnsembleID & Chromosome \\
\hline Ifi204 & ENSMUSG00000073489 & 1 \\
\hline Ifi202b & ENSMUSG00000026535 & 1 \\
\hline Exo1 & ENSMUSG00000039748 & 1 \\
\hline Gbp5 & ENSMUSG00000105504 & 3 \\
\hline Gbp7 & ENSMUSG00000040253 & 3 \\
\hline Gbp3 & ENSMUSG00000028268 & 3 \\
\hline Gbp2 & ENSMUSG00000028270 & 3 \\
\hline Timp4 & ENSMUSG00000030317 & 6 \\
\hline Tmem40 & ENSMUSG00000059900 & 6 \\
\hline Ctla2a & ENSMUSG00000044258 & 13 \\
\hline S1pr3 & ENSMUSG00000067586 & 13 \\
\hline Drd1 & ENSMUSG00000021478 & 13 \\
\hline Cdhr2 & ENSMUSG00000034918 & 13 \\
\hline Catsper3 & ENSMUSG00000021499 & 13 \\
\hline Nlrp4f & ENSMUSG00000032999 & 13 \\
\hline Zfp953 & ENSMUSG00000098905 & 13 \\
\hline Lpcat1 & ENSMUSG00000021608 & 13 \\
\hline Erap1 & ENSMUSG00000021583 & 13 \\
\hline$F 2 r$ & ENSMUSG00000048376 & 13 \\
\hline Conb1 & ENSMUSG00000041431 & 13 \\
\hline Itga2 & ENSMUSG00000015533 & 13 \\
\hline SIc25a1 & ENSMUSG00000003528 & 16 \\
\hline lgf2bp2 & ENSMUSG00000033581 & 16 \\
\hline Rtp4 & ENSMUSG00000033355 & 16 \\
\hline Apod & ENSMUSG00000022548 & 16 \\
\hline Kif20a & ENSMUSG00000003779 & 18 \\
\hline$M z b 1$ & ENSMUSG00000024353 & 18 \\
\hline SIc4a9 & ENSMUSG00000024485 & 18 \\
\hline Fchsd1 & ENSMUSG00000038524 & 18 \\
\hline
\end{tabular}

Figure 6. Subclustering of the $\mathrm{m} 5$ immune module identifies five subclusters with immune function and QTLs that map to immunologically relevant genetic regions. (A) Composition of $\mathrm{m} 5$ based on subcluster (SC) gene membership; for the 848 genes in $\mathrm{m} 5$ the subclusters broke out into 291 genes in SC1, 228 genes in SC2, 176 genes in SC3, 108 genes in SC4, and 45 genes in SC5. (B) Spearman correlations (adj. $P<0.05)$ of $\mathrm{m} 5$ and $\mathrm{SC} 1-\mathrm{SC} 5$ to short-term and long-term memory measures. $(C, D)$ The proportion of variance in measures for short-term memory $(C)$ and long-term memory $(D)$ explained by $\mathrm{m} 5$ (dark red) and SC1-SC5 (light red) average expression. For B-D SCs are denoted by the most significantly enriched $\mathrm{GO}$ term. ( $E$ ) Genetic mapping of the first principal component (PC1) as module eigengene for SC5; Age, sex, and FAD mutation status were used as additive covariates. $(F)$ Table denoting proteincoding genes symbols, ensemble IDs, and chromosomal location for each gene within the SC5 QTL peaks that were also $\mathrm{m} 5$ members.

significant module-A $\beta 1-42$ interactive effects on cognitive outcomes that identified only one module-A $\beta 1-42$ relationship (m4) had a significant interactive effect on Y-maze \%SA (Supplemental Table S8). Since only a single interactive effect was obtained, we tested the additive effect of module expression and cortical A $1-42$ accumulation using Type 2 ANOVA statistical tests (Supplemental Table S8), as well as likelihood-ratio (LRT) tests to determine if the addition of amyloid into the model significantly improved the linear fit that explained cognitive outcomes using module expression (Satorra and Saris 1985). Within AD-BXD individuals, module expression for each significantly correlated module explained up to $10 \%$ of the variance in Y-maze working memory \%SA outcomes, and the models for this cognitive outcome were not significantly improved by the addition of A $\beta 1-42$ ac-

cumulation (Fig. 7D). This was similar for CFA short-term memory outcomes, except the relationship of $\mathrm{m} 6$ to CFA slope was significantly improved by the addition of A $1-42$ accumulation into the linear model (Fig. 7E). One thing to note is that the effect of $\mathrm{m} 7$, the module upstream of working memory and shortterm memory in the Bayesian model (Fig. 4), on cognitive outcomes was not improved by the addition of A $\beta 1-42$ accumulation to the linear model, suggesting a nominal role for how amyloid influences these module-trait relationships. Finally, for CFM long-term memory outcomes, the effect of all except for two modules (m4, m7) was significantly improved by the addition of A $\beta 1-42$ into the linear model, suggesting that amyloid may play a more direct role on influencing the module-trait relationships for modules associated with long-term memory (Fig. 7F). Additionally, all except one of the m5 SCs were significantly improved by the addition of AB1-42 into the linear model (Supplemental Fig. S5A, $B)$, suggesting that the specific relationship between $\mathrm{m} 5$ (and subsequent SCs) and long-term memory outcomes are influenced by amyloid accumulation. To gain a further understanding of the age-related factors that could drive these relationships, we recreated linear models within 6 mo AD-BXDs and 14 mo AD-BXDs (Supplemental Fig. S6). Interestingly, while the majority of the variance for working memory and shortterm memory outcomes were observed within the 6 mo AD-BXD cohort, module expression explained more variance in 14 mo AD-BXDs for long-term memory outcomes (Supplemental Fig. S6C-E). This indicates that early-in-life perturbation of $\mathrm{m} 7$ at 6 mo or earlier may have a greater effect on altering working and short-term memory cognitive domains, while perturbation of $\mathrm{m} 5$ at a later age (14 mo) may prove more successful to improve the long-term memory cognitive domain. Together, these findings suggest a specific role of the interaction of cortical A $\beta 1-42$ accumulation and module expression on influencing long-term memory outcomes, while a more indirect or nominal role of A $\beta 1-42$ accumulation on influencing how modules affect working memory and short-term memory outcomes.

\section{Cognitively relevant mouse modules replicate in aged human molecular brain networks}

A criticism of using mouse models for $\mathrm{AD}$ research is that many previous findings in mouse models have failed to translate to human patients. Therefore, we tested the replication of all mouse coexpression modules in human brain data, including those predicted to be upstream of variation in cognitive outcomes across our mouse population. Using a consensus genetic clustering analysis of dorsolateral prefrontal cortex (DL-PFC) from aged individuals (AD and 
A

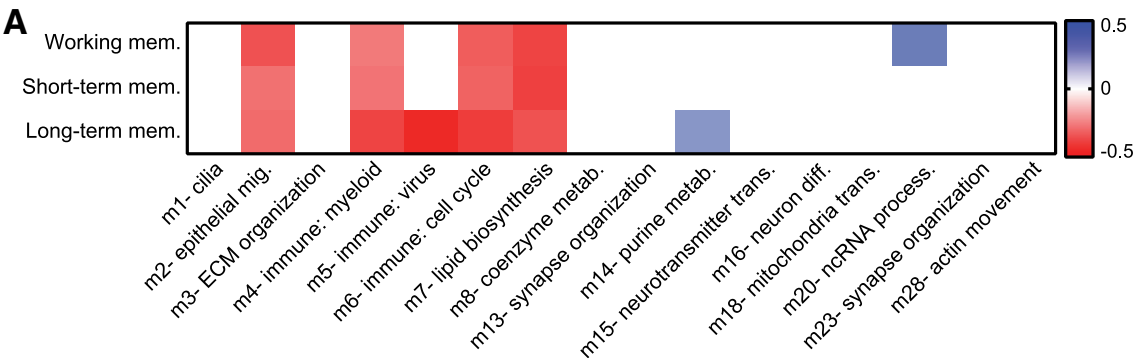

B

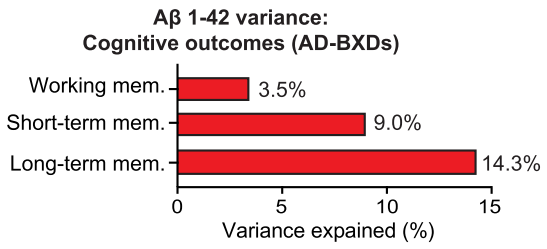

C

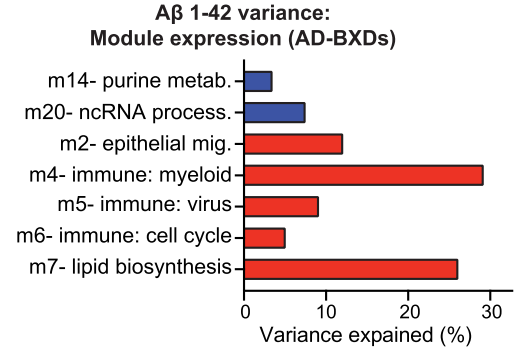

D

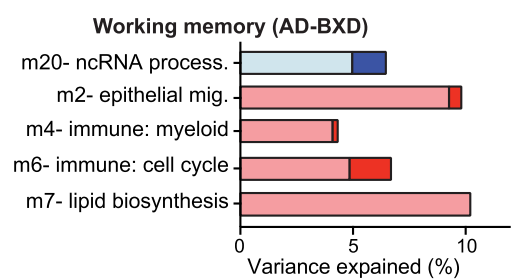

Figure 7. $A D-B X D$ long-term memory module-trait relationships are impacted by the amount of amyloid accumulation. (A) Average standardized module expression for 5XFAD mice was correlated to cognitive traits to identify modules that significantly modify cognitive function in the AD-BXD mouse population. Only the modules that were significantly correlated with cognitive function in the entire population were tested for significant relationships within the AD-BXD cohort. Modules with no significant correlation to corresponding cognitive outcome are depicted in white. (B) The proportion of variance in cognitive outcomes explained by amount of cortical amyloid (quantified by A $31-42$-specific sandwich colorimetric ELISA) in AD-BXD mice. (C) The proportion of variance in module expression explained by amount of cortical $A \beta 1-42$. $(D-F)$ The proportion of variance in measures for working memory $(D)$, short-term memory $(E)$, and long-term memory $(F)$ explained by module expression and amount of cortical AB1-42 in AD-BXD mice, using an additive Type 2 ANOVA model. Variance explained by individual modules significantly positively (blue) and negatively (red) correlated to corresponding cognitive trait are in lighter colors, while amount of variance explained by amyloid as part of the additive linear model is in darker colors. Likelihood-ratio (LRT) tests were performed for each model to test if the addition of cortical A $1-42$ into the additive model generated a better fit of linear model. Significant $P$-values $(P<0.05)$ of LRT tests are denoted by black asterisks, indicating the addition of cortical $A \beta 1-42$ to the model provides a better linear fit than module expression alone. All modules are annotated by the most significantly enriched $\mathrm{GO}$ term (NS GO = no significant GO enrichment).

non-AD) (Mostafavi et al. 2018), we performed statistical hypergeometric tests to see how well our mouse modules overlapped with those found in humans. We found that the majority $(15 / 16)$ of the cognitively relevant mouse modules were significantly (adj. $P$ $<0.05$ ) conserved in human DL-PFC modules (Fig. 8). The only nonconserved module was $\mathrm{m} 6$ (immune/cell cycle), which was surprising since indicators of inflammation are consistently observed in $\mathrm{AD}$ tissue. However, other immune-related modules were conserved, while the lack of conservation for this module may be due to its mixture of innate immune response genes and cell cycle genes. We also tested the conservation of molecular networks generated by a meta-analysis of multiple brain regions from human aged and AD tissue (Wan et al. 2020), and found that all 16 significantly correlated mouse modules in at least one brain gene network (Supplemental Fig. S7). Specifically, all of the significantly correlated mouse modules were conserved in the DL-PFC molecular networks from the Accelerating Medicines PartnershipAlzheimer's Disease (AMP-AD) consortium meta-analysis molecular networks, strengthening our findings from the conservation with the Mostafavi DL-PFC networks. Notably, the two prioritized modules from our Bayesian analysis (m5 and $\mathrm{m} 7$ ) were both significantly conserved with several human modules associated with cognitive function across the population (Fig. 8; Supplemental Fig. S7). These results highlight that both the major molecular networks and ontology pathways observed in a genetically diverse mouse population are also replicated in human cohorts, making our current findings prioritizing $\mathrm{m} 5$ and $\mathrm{m} 7$ relevant to human $\mathrm{AD}$ and aging populations.

\section{Discussion}

Systems genetics identifies causal module-trait relationships in a population of $A D$ mice that explain individual differences in cognitive

\section{outcomes}

The search for the underlying genetic and molecular causes of AD cognitive decline has been the primary goal for identifying and developing therapeutics for several decades (Graham et al. 2017). Identification of such genetic and molecular mechanisms that influence $\mathrm{AD}$ symptoms using preclinical mouse models has been criticized because of the poor translatability of findings in mouse models to effectively treating human $\mathrm{AD}$ (Onos et al. 2016; Drummond and Wisniewski 2017). Modeling the disease in more genetically diverse mice may be one avenue to improve the construct validity of preclinical models (Hyman and Tanzi 2019; Neuner et al. 2019a). Using this model, we were able to leverage transcriptomic and phenotypic information from pre- and postsymptomatic mice with and without AD mutations to identify the molecular networks that are predicted to directly influence variation in cognitive outcomes across a population. We found many sets of coexpressed genes, each representing the activity of one or more molecular systems, that were significantly correlated with population-wide differences in cognition, and explained up to $30 \%$ of the variance in cognitive outcomes. This is important because while high-impact variants 


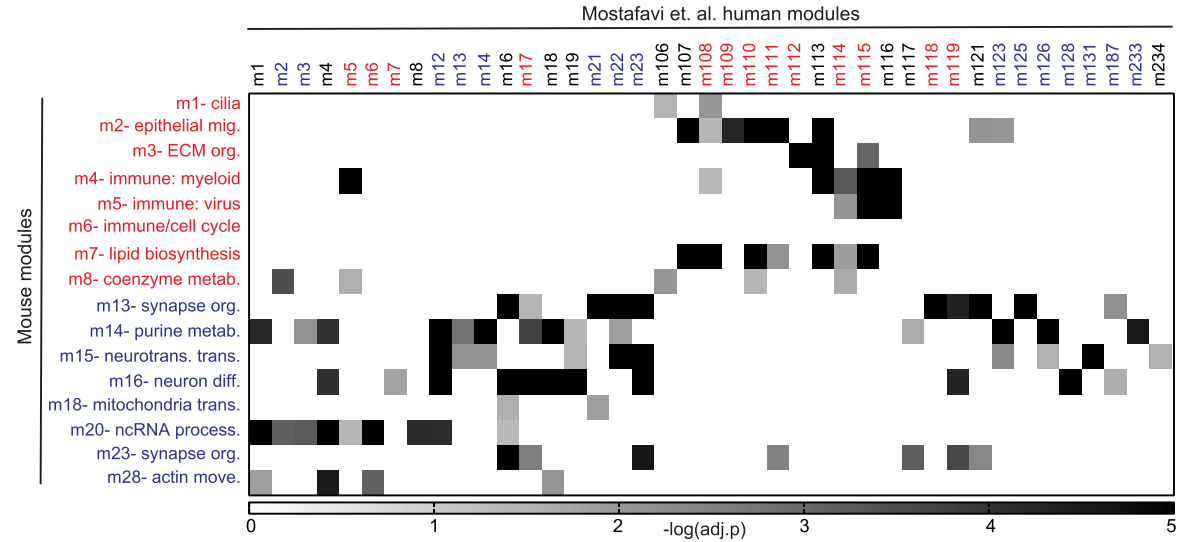

Figure 8. Mouse modules significantly associated with cognitive outcomes have high degree of conservation to human AD modules. Mouse modules significantly correlated to cognitive measures (left, $y$-axis) were examined for gene conservation in previously established (Mostafavi et al. 2018) aged/ AD human dorsolateral prefrontal cortex (DL-PFC) modules (top, $x$-axis). Modules are colored based on positive (blue) or negative (red) correlation to corresponding mouse (working/short-term/long-term memory) or human (slope of decline) cognitive measure. Heatmap depicts degree (measured by -log [adj. P-value]) of overlap (hypergeometric test) between human and mouse modules. Only significant (adj. P-value $<0.05$ ) overlaps are depicted, while nonsignificant overlaps are white. Human modules were excluded if they did not overlap with at least one mouse module tested above. Mouse modules are denoted by the most significantly enriched GO term.

such as those in APOE can explain a considerable proportion of $\mathrm{AD}$ risk, many of the more common $\mathrm{AD}$ variants have much smaller effect-sizes making new gene targets less impactful for translation to larger populations (Bertram et al. 2007; Genin et al. 2011; Mostafavi et al. 2018). In contrast, the upstream molecular networks identified here explain larger variances and may have a much larger effect on improving cognitive outcomes on the population levels. The networks consistently explaining the most variance were enriched for viral and myeloid immune pathway genes (m4, m5, $\mathrm{m} 6)$, as well as lipid and coenzyme metabolism pathway genes $(\mathrm{m} 7, \mathrm{~m} 8)$. Additionally, genes within these biologically significant modules were found to be primarily expressed in nonneuronal celltypes such as astrocytes and microglia, suggesting that regulation of the specific pathways [lipid biosynthesis, IL-1 signaling, interferon (IFN) signaling] within these cell-types contributes most significantly to divergent cognitive outcomes across resilient and susceptible individuals (Zhang et al. 2014). These observations were further substantiated by our Bayesian modeling of moduletrait relationships in that the only modules directly upstream of cognitive outcomes were enriched for nonneuronal genes: the astrocyte lipid biosynthesis module $(\mathrm{m} 7)$ for working and short-term memory, and the microglia viral immune module (m5) for longterm memory. This model proposed two cell-type enriched molecular networks to prioritize for further investigation, since our model predicted that population-wide differences in these networks have greatest direct impact on regulating cognitive outcomes.

\section{Targeting astrocyte lipid biosynthesis and IL-1 signaling may promote resilience to working memory and short-term memory decline}

The gene network that our model found to be directly upstream of working and short-term memory was $\mathrm{m} 7$, which was enriched for "lipid biosynthesis" GO biological pathways. Notably, m7 was exclusively enriched for genes found in astrocytes, specifically, implicating astrocytic lipid biosynthesis as critical for determining cognitive outcomes. Further IPA analysis also highlighted a notable $\mathrm{m} 7$ gene, Rhoa, that emerged in three of the five top enriched canonical pathways (Table 1). This gene is of particular interest because therapeutically targeting RhoA pathways has been found to be promising for improving $\mathrm{AD}$ and mixed dementia symptoms, proposing a method by which targeting $\mathrm{m} 7$ could influence AD cognitive outcomes (Herskowitz et al. 2013; Gentry et al. 2016; Henderson et al. 2019). While these previous studies have investigated RhoA pathway inhibition in the context of amyloid and tau pathology in $\mathrm{AD}$ and mixed dementia, these findings could suggest that inhibition of $\mathrm{m} 7$ via the RhoA pathway could also improve cognitive outcomes. Additionally, our IPA analysis identified other pathways of interest, such as "IL-1 signaling," and "cholesterol biosynthesis." IL-1 signaling in astrocytes plays an important role in promoting inflammation. IL-1 signaling deficiency has also been demonstrated to have detrimental effects on hippocampal synaptic plasticity (Moynagh et al. 1994; Ben Menachem-Zidon et al. 2011). As the IL-1 signaling pathway is also an indicator of inflammation, the overall representation of this module could be for reactive (or A1) astrocytes, which have been widely described as being induced by inflammatory insult (Zamanian et al. 2012; Anderson et al. 2016; Liddelow et al. 2017). More specifically, reactive astrocytes are thought to be induced by proinflammatory cytokines TNF $\alpha$, IL-1, and C1Q, which are highly expressed in microglia in the CNS (Zhang et al. 2014; Bennett et al. 2016). Two of the three transcripts for these cytokines appear in $\mathrm{m} 5$ (Tnf, Il1a), suggesting an interplay between these two modules and representative cell-types that directly influence cognitive resilience. Finally, an indicator of inflammation that has been characterized in astrocytes and microglia is the formation of lipid droplets within these cells, which synthesizes with our other finding of additional enrichment of lipid metabolism pathway genes in this module (Farmer et al. 2019; Marschallinger et al. 2020).

In addition to the interesting proinflammatory pathways, the "cholesterol biosynthesis" pathway present in $\mathrm{m} 7$ suggests an additional mechanism by which astrocytes may dictate cognitive outcomes. Cholesterol biosynthesis has been previously implicated in $\mathrm{AD}$ pathogenesis due to a variety of mechanisms. For example, cholesterol influences the relationship between amyloid precursor protein (APP) and in the production of $A \beta$, but the mechanism by which this occurs is still not understood well (Shobab et al. 2005). In addition, cholesterol biosynthesis is of particular importance to mature neurons since astrocytes are the primary cholesterol source for mature neurons in the CNS (Poirier et al. 1993; Pfrieger 2003). A negative association between lipid biosynthesis and cognitive outcomes could indicate a stall or dysfunction in this process by either depriving neurons of necessary lipids or over-production within astrocytes. Finally, APOE- the most significant risk factor for sporadic $\mathrm{AD}$ - is also intimately involved in cholesterol metabolism. Risk variants in $A P O E$ have been hypothesized to disrupt the ability of APOE to bind cholesterol and therefore contribute to $\mathrm{AD}$ pathogenesis (de Chaves and Narayanaswami 2008; Wood et al. 2014). As the BXD panel contains naturally occurring variation in the Apoe receptor binding domain, this study may have identified the cell-type and pathway by which Apoe influences cognitive resilience (Arboleda-Velasquez et al. 2019; Neuner et al. 2017, 2019a). Further studies will be conducted to (1) test if lipid biosynthesis is higher in astrocytes from mice that perform 
worse on short-term and/or working memory cognitive tasks using pulse-chase experiments (Rambold et al. 2015; Thiele et al. 2019), and (2) observe if the IL-1 inflammatory signature from this module synthesizes with lipids by evaluating lipid droplet formation within astrocytes from AD-BXD individuals using BODIPY staining (Li et al. 2020). These experiments will further our understanding of the contribution of specific pathways we believe to be involved in astrocytes that help dictate $\mathrm{AD}$ cognitive outcomes.

\section{Fgf2 as a possible regulator of working and short-term memory outcomes}

In this study we leveraged the power of the known genetic composition of our AD-BXD panel to identify a chromosomal region, and a protein-coding gene within, that regulates the expression of $\mathrm{m} 7$, the module directly upstream of Y-maze working memory and CFA short-term memory outcomes. The nominated gene, Fgf2, showed a strong negative overall relationship of expression with short-term memory outcomes. The FGF2 protein has a variety of known functions in the CNS, including promoting angiogenesis, neurogenesis, and improving symptoms of cognitive aging (Woodbury and Ikezu 2014). FGF2 is primarily expressed in astrocytes in the adult brain, but can also be secreted by neurons when damaged to produce a distress signal to recruit microglia and astrocytes to the site of damage (Reuss and von Bohlen und Halbach 2003; Noda et al. 2014). Functionally, FGF2 is known to regulate the proliferative response to oligodendrocyte precursor cells (OPCs) during remyelination of axons after acute demyelination injury, suggesting another role of FGF2 signaling in regulating glia-neuron homeostasis (Frost et al. 2003; Magy et al. 2003; Murtie et al. 2005). Experimentally induced spinal cord and CNS injury has shown to promote reactive astrogliosis, and these reactive astrocytes have been previously shown to up-regulate FGF2, suggesting an additional involvement of FGF2 in the proliferation and reactivity of astrocytes to sites of injury and recovery (Fahmy and Moftah 2010). Increased FGF2 (or basic FGF, bFGF) has been observed in reactive astrocytes and the neurons surrounding amyloid plaques, as well as its localization to neurofibrillary tangles and cerebrovascular deposits in AD patient brain tissue (Gomez-Pinilla et al. 1990; Stopa et al. 1990; Cummings et al. 1993). However, delivery of FGF2 into the hippocampus of APP mice using AAV2/1 viral construct caused mice to show enhanced long-term potentiation, reduced $A \beta$ production, and enhanced neurogenesis, suggesting FGF2 can act to halt AD pathophysiology (Kiyota et al. 2011). Together, while our data suggest that more $F g f 2$ is detrimental to cognitive performance, other data suggest FGF2 activity is important to glial and neuronal response to injury or pathological environment. However, characterization of bFGF with AD pathology suggests it could be part of the reactive astrocyte response to disease activity. Further work will have to be conducted to determine the cell-type for which $F g f 2$ is promoting a negative correlation with cognitive outcomes, the cellular pathways by which it is acting, and how it could be detrimental to AD cognitive outcomes.

\section{Targeting viral and adaptive immune pathways to promote long-term memory resilience}

The robust associations of microglia and microglial processes with improving cognitive outcomes in mice is consistent with genetic (Lambert et al. 2013; Kunkle et al. 2019) and cellular data (Sosna et al. 2018; Spangenberg et al. 2019), indicating their relevance to AD disease activity and cognition (Hong et al. 2016; Kinney et al. 2018). Surprisingly, when we interrogated our molecular networks in search of known AD risk genes enriched in immune processes, we found that the majority of the sporadic $\mathrm{AD}$ immune-related risk genes were actually present in $\mathrm{m} 4$, our im- mune: myeloid module, and not m5, our immune: virus module. It was interesting that several high impact sporadic $\mathrm{AD}$ genes (BIN1, TREM2, APOE) were present in a single module (we allowed discrete assignment of each gene to one module in this study), and also that this module did not explain as much variance in cognitive outcomes as the viral immune pathway, with m4 explaining $6.6 \%$ and $\mathrm{m} 5$ explaining $10.5 \%$ of the variance in long-term memory (Fig. 3). It could be possible that these m4 LOAD genes and immune: myeloid pathways in $\mathrm{m} 4$ have a more indirect effect on $\mathrm{AD}$ cognitive resilience as a molecular system, compared to the viral immune response pathway.

Given recent evidence that viral immune pathways, including the type-1 interferon (IFN) pathway, are up-regulated in human AD patients, the viral immune network identified in $\mathrm{m} 5$ is an interesting pathway associated with the neuroinflammatory response observed in AD (Taylor et al. 2014). Previous studies using mouse models of $\mathrm{AD}$ have demonstrated that knockout of the type- 1 IFN receptor (IFNAR), an important receptor for the viral immune response, causes reduced amyloid monomer levels, and partial rescue of performance on the Morris water maze task at 9 mo of age (Minter et al. 2016). Recently, conflicting evidence has emerged about the implications of viral infections, such as the presence of herpesviruses (HSVs, HHVs) in postmortem brain tissue, with AD cognitive deficits (Allnutt et al. 2020; Readhead et al. 2018). While these infections would not be present in the mice used in this study, since they are raised and aged in a controlled lowpathogen environment, it is interesting that we still observe activation of antiviral pathways. Additionally, we found very little overlap in $\mathrm{m} 5$ genes with genes that drive network activity of cells that are infected with herpesviruses such as HSV-1 or HHV6A found in AD patient brains (Readhead et al. 2018; Drayman et al. 2019). This suggests that the networks activated by these viruses in human patients are independent of the viral immune pathways enriched in $\mathrm{m} 5$, and that the $\mathrm{m} 5$ network represents an immune response to nonviral pathological burdens present in the brain, such as $A \beta$. This is supportive of our finding that the effect of $\mathrm{m} 5$ on long-term memory outcomes was improved by the addition of cortical A $\beta$ accumulation in the linear model. However, since the mice used in this study have not been exposed to a systemic viral challenge, it could be possible that activating antiviral pathways systemically, by either HSV infection or poly I:C challenge, could activate other molecular networks that explain more variance in long-term memory outcomes compared to those activated in mice raised in a sterile environment. More work needs to be done in this field to decipher a specific interplay between environmental factors (such as viral exposomes and diet), amyloid and the relevance of their interaction in AD pathogenesis (Dunn et al. 2019).

Along with viral immune pathways being represented in $\mathrm{m} 5$, our subclustering analysis highlighted more distinct immune pathways that continued to be correlated with long-term memory measures (Fig. 6; Supplemental Fig. S5). The enriched pathways revealed by these subclusters further supported the importance of viral immune response pathways (represented in SC1), as well as indicators of an adaptive immune response (lymphocyte differentiation represented in SC2), and microglial migration (represented by IL-8 signaling in SC4). These pathways were interesting because while the importance of viral immune pathways remained, we also identified other pathways such as the IL-8 signaling pathway that we would not have identified as the top enriched pathway in the large $\mathrm{m} 5$ module. The IL- 8 signaling pathway is interesting in the context of $\mathrm{AD}$ because IL-8 (with the official gene symbol of $\mathrm{Cxcl8}$ ) is known to induce microglial migration, and has been previously found to increase in microglia exposed to amyloid (Lue et al. 2001; Franciosi et al. 2005). Additionally, IL-8 has been proposed as a biomarker for AD cognitive impairment and cerebrovascular dysfunction when it was found to be up-regulated in blood 
serum of $\mathrm{AD}$ and cognitively impaired individuals with cerebrovascular disease compared to noncognitively impaired counterparts (Zhu et al. 2017). Therefore, enrichment of this pathway could be a possible indicator of population-wide differences in microglial amyloid migratory response, and differences in cerebrovascular dysfunction across the AD population. We also may have identified a measurable marker of resilience and susceptibility to cognitive impairment by identification of this $\mathrm{m} 5$ subcluster which we will test in future studies for validation. Finally, subclustering of $\mathrm{m} 5$ also allowed for genetic mapping of the SC5 eigengene to a region on chromosome 1 within the AD-BXD population (Fig. 6E,F; Supplemental Fig. S5C,D). This region was interesting because several genes within this locus were also m5 members, and associated with IFN signaling (Ifi204, Ifi202b), adaptive immune response (Exo1, Fcrl6), and a glia-specific potassium channel, Kcnj10 (also known as Kir4.1). The region identified on chromosome 1 is incredibly interesting because of its enrichment for these glia and immune-related genes that were also m5 members, as well as inspection of these genes with the Sanger Mouse Genomes database reveals several coding variants between $\mathrm{C} 57 \mathrm{BL} / 6 \mathrm{~J}$ and DBA/2J mice (Keane et al. 2011). Additionally, SC5 was only significantly correlated with long-term memory outcomes, suggesting a specific role of this SC on integrating overall $\mathrm{m} 5$ activity with individual differences in long-term memory outcomes. Future work will elucidate how this SC5 molecular network, the chromosome 1 QTL, and the genes within it possibly control overall $\mathrm{m} 5$ activity and longterm memory outcomes.

Overall, our data and suggest that the specific pathways that will have the greatest impact on improving cognitive outcomes are those involved in the microglial viral immune and migratory response. It is still unclear if this module is representative of the local brain immune response by activation of microglia, or if this pathway is also comprised of infiltrating immune cells that can also contribute to the neuroinflammatory response in later stages of $\mathrm{AD}$, which was proposed by the enrichment of lymphocyte differentiation genes in m5 SC2 (Heppner et al. 2015). Additionally, the distinction between peripheral and local neuro-immune responses in the brain are difficult since microglia and peripheral macrophages express many of the same marker genes and proteins. Although our Bayesian model has found that the neuroinflammatory response is the major contributor to long-term memory resilience in our aging model, further experiments will be done to distinguish how much of this resilience network is dictated by peripheral or local innate immune responses. This will include investigating the role of the systemic immune system, and how increased infiltrating systemic immune cells (CD4+ T cells, B cells, peripheral macrophages) may play a negative role in cognitive resilience by evaluating brain gene and protein expression of these peripheral markers in this mouse population and comparing to TMEM119+ microglia. Specifically, we hypothesize from these data that inhibition of the type-1 IFN signaling pathway in more susceptible mice will promote cognitive resilience by delaying the neuroinflammatory response that causes cognitive decline in AD. Additionally, we will further investigate the role of IL-8 as a possible biomarker of neuroinflammatory activity in susceptible AD individuals. Finally, we have proposed several gene candidates from QTL mapping of the m5 SC5 eigengene that we will examine as possible targets to promote resilience to $\mathrm{AD}$ long-term memory deficits.

\section{Elucidating the contribution of $A \beta$ accumulation to the impact of molecular networks on cognitive outcomes}

As we have stated previously, AD is classified by progressive and severe memory decline, as well as the pathological presence of beta-amyloid (A $\beta$ ) plaques and neurofibrillary tau tangles (Selkoe
1991). It is still widely debated if amyloid is directly involved in determining $\mathrm{AD}$ cognitive trajectories, or if it is more of associated pathology with limited involvement in cognitive decline (Murphy and LeVine 2010). We previously found in our AD-BXD population that while cortical A $\beta 1-42$ levels were variable across genetic backgrounds (especially at $14 \mathrm{mo}$ ), this variability did not directly correlate with cognitive function (Neuner et al. 2019a). Here, we found that many of our identified module-trait relationships were strengthened when we isolated and tested these relationships in the AD-BXD population (Supplemental Table S4; Fig. 7A), indicating the presence of FAD mutations is necessary for these molecular networks to influence cognitive outcomes. Using additional linear modeling, we tested the effects of cortical A $\beta 1-42$ accumulation on module expression, and if it could improve the influence of module expression on cognitive outcomes. Our results indicated a domain-specific effect that working memory and short-term memory were primarily influenced by module expression, and largely independent of individual differences in amyloid burden (Fig. 7D,E). However, for long-term memory cortical A $\beta 1-42$ accumulation improved the effect of module expression on memory outcomes, indicating an indirect role of amyloid on cognition through influencing module expression (Fig. 7F; Supplemental Fig. S5B). Therefore, we hypothesize that the role of $\mathrm{A} \beta$ on $\mathrm{AD}$ cognitive trajectories could be more complex than initially anticipated, and dependent on the cognitive domain and molecular pathway. It is possible that more module variance could be explained if we had measured and compared hippocampal AB1-42 accumulation to gene expression changes instead of cortical A $\beta 1-42$, and are working to test this difference in future studies using A $\beta 1-42$ staining of fixed hemibrains from the same mice investigated here. Further studies will also test this model to see how perturbation of certain molecular networks influences cognitive outcomes, and if these networks will alter amyloid accumulation or if secondary amyloid targeting is also needed to have maximum effect, especially for long-term memory outcomes.

\section{Advantages of diverse mice for defining the genetic origins of resilience}

In this study, we were able to leverage previously generated transcriptome and phenotype information from a population of genetically diverse mice to identify the molecular networks that directly influence population-wide differences in cognitive outcomes. An advantage of using a genetic reference panel such as the BXDs is that we could leverage the well-defined genetics of this population to perform genetic mapping that anchored many of our moduletrait relationships.

In addition to identifying immune and lipid metabolism pathways, as well as the $F g f 2$ gene, as being possible direct regulators of AD cognitive outcomes, this study also identified neuronal and synaptic modules that were correlated to short-term memory outcomes (see Fig. 3A). We wanted to highlight these neuronal pathways in particular because recent studies using $\mathrm{AD}$ patient brain tissue have found that changes in dendritic spine morphology can indicate resilience to AD cognitive decline (Boros et al. 2017, 2019). Further studies will test the importance of neuronal pathways in providing $\mathrm{AD}$ resilience, especially in the context of susceptible compared to resilient microglia or astrocyte environments, since targeting neuronal pathways have also been suggested to be desirable for therapeutic intervention in AD (Gentry et al. 2016; Henderson et al. 2019). Future work overlaying gene expression from mice that receive such interventions will also highlight how these molecular networks interact with each other when a specific module or target is perturbed. Finally, analysis of gene expression from this model system and comparison to human brain networks has nominated conserved molecular systems that are 
relevant to diverse $\mathrm{AD}$ cognitive outcomes. The identification of these relevant molecular systems will lead to a better understanding of preclinical models of human $\mathrm{AD}$, which will allow for testing of novel resilience-based therapeutic strategies to improve $\mathrm{AD}$.

\section{Materials and Methods}

\section{Bioethics}

All mouse experiments occurred at University of Tennessee Health Science Center and were carried out in accordance with the principals of the Basel Declaration and standards of the Association for the Assessment and Accreditation of Laboratory Animal Care (AAALAC), as well as the recommendations of the National Institutes of Health Guide for the Care and Use of Laboratory Animals. The protocol was approved by the Institutional Animal Care and Use Committee (IACUC) at the University of Tennessee Health Science Center.

\section{Animals}

All data used in this study are from a mouse model of human AD, the AD-BXD panel, which were previously described (Neuner et al. 2019a). Briefly, female B6 mice hemizygous for the 5XFAD transgene (B6.Cg-Tg(APPSweF1LonPSEN1*M146L*L286V)6799Vas/ Mmjax, Stock No. \#24848-JAX) were mated to males from the BXD genetic reference panel (Williams et al. 2001; Peirce et al. 2004). One generation of breeding B6-5XFAD dams with BXD sires results in a set of 26 isogenic F1 AD-BXD strains that either harbor the 5XFAD transgene or are nontransgenic (Ntg)-BXD littermate controls. Male and female AD-BXD mice were group housed as a mix of 5XFAD and nontransgenic same-sex littermates and maintained on a $12 \mathrm{~h}$ light-dark cycle.

\section{Y-maze}

Behavioral testing to assess mice for working memory function was performed as previously described (Neuner et al. 2019a). In brief, mice were habituated to transport and to the testing room for $3 \mathrm{~d}$ prior to testing. The y-maze test of spontaneous alternation was performed for 8 min using a clear acrylic Y-shaped maze with arms that were 2 inch wide $\times 12$ inch long $\times 12$ inch high (Oakley et al. 2006; Neuner et al. 2019a). The maze was placed on a table in a dimly lit room and spatial cues were displayed on walls around the table. Mice were placed in a randomized start arm and video tracking software was used to monitor arm entries (ANY-maze, Stoelting Co.). An arm entry was called when the mouse's entire body, including the two back feet, entered the arm. The sequence and total number of arms entered was recorded, and the percentage of successful alternations was calculated as follows: number of alternations/maximum possible alternations (total number of arms entered -2$) \times 100$. Mice were evaluated for performance on this task longitudinally every 2 mo until their specified harvest age (6 mo or $14 \mathrm{mo}$ ). AD-BXD and Ntg-BXD strain/age/sex specific group averages were generated for further downstream analyses.

\section{Contextual fear conditioning}

After $3 \mathrm{~d}$ of habituation to transport and to the testing room, mice underwent contextual fear conditioning (CFC) as previously described (Neuner et al. 2015). On the first day of CFC training, mice were placed in a chamber and four $0.9 \mathrm{~mA} 1 \mathrm{sec}$ (s) foot shocks were delivered after a baseline period. Over the period of $700 \mathrm{sec}$ four foot shocks were delivered, and the postshock intervals were defined as the $40 \mathrm{sec}$ following the offset of each foot shock. The slope of the line formed when the percentage of time spent freezing following each shock was calculated as a function of each interval was used as a measure for contextual fear acquisition (CFA), and short-term memory. Twenty-four hours after training, mice were placed back into the training chamber and the percentage of time spent freezing throughout the entire $600 \mathrm{sec}$ test was measured as an index of contextual fear memory (CFM), and long-term memory. AD-BXD and Ntg-BXD strain/age/sex specific group averages were generated for further downstream analyses.

\section{RNA sequencing}

Initial RNA sequencing (RNA-seq) of hippocampus from the AD-BXD panel [6 mo, $n=71$ (47 females/24 males) and 14 mo, $n=86$ (45 females/41 males)], and Ntg-BXD counterparts [6 mo, $n=31$ (17 females $/ 14$ males) and $14 \mathrm{mo}, n=33$ (17 females/16 males)] has been previously reported ((Neuner et al. 2019a) GEO accession number GSE101144; (Neuner et al. 2019b) GEO accession number GSE119215). Here, we expand upon this data set to include RNA sequencing data from an additional 71 Ntg-BXD mice, 27 of which were 6 mo of age ( 22 females $/ 5$ males) and 44 of which were 14 mo of age (28 females/16 males, GEO accession number GSE119408). RNA-seq was performed as previously described (Neuner et al. 2019a). Briefly, all samples were isolated using the Qiagen RNeasy Mini kit, libraries were prepared using Truseq Stranded mRNA Sample Preparation Kit (Illumina Inc.), and sequenced by 75 bp sequencing on an Illumina HiSeq2500. Because sequencing was performed on mice harboring alleles for C57BL/6J (B6) and DBA2/J (D2), the GBRS/EMASE pipeline (Raghupathy et al. 2018) developed by the Churchill group at The Jackson Laboratory was used in order to align reads to the correct allele, allowing for quantification of both total reads for a gene and reads assigned to the $\mathrm{B} 6$ or D2 allele. To account for the expression of the 5XFAD transgene, RNA-seq reads were additionally aligned to the mutated human APP and PSEN1 sequences. Genes were filtered to require an average of at least 1 transcript per million (TPM) in $50 \%$ of samples. RNA data was batch corrected using ComBat (Johnson et al. 2007; Leek et al. 2012), and biological replicates were averaged together for downstream analyses. During batch correction, two samples (S3 and S285) were included in data upload, but dropped in final analyses as outliers before strain averaging. For final downstream analysis, individual samples belonging to the same BXD strain/sex/age/mutation carrier group were averaged to generate 156 distinct groups.

\section{Formation of gene coexpression modules}

Consensus clustering was used on gene transcripts per million (TPM) values from the RNA-seq results using a novel algorithm (Gaiteri et al. 2015) to identify groups of coexpressed genes or "modules" that represented genes that were commonly regulated together across the examined mouse population. The consensus clustering algorithm, SpeakEasy, was used to identify 31 discrete transcriptional modules of coexpressed genes that ranged in size between four to 1419 gene members. Twenty eight of the 31 modules contained more than 29 gene members, and could be assigned for $>99 \%$ of the genes analyzed for coexpression. Therefore, three modules with fewer than 30 genes were excluded from downstream analysis, and contained $<1 \%$ of total genes used for coexpression. This is consistent with previous methods for exclusion of modules with small membership (Mostafavi et al. 2018).

\section{Module functional enrichment}

Gene Ontology (GO) functional enrichment analysis was performed on the genes within each module using the clusterprofiler package in R. GO categories with a hypergeometric enrichment adjusted $P<0.05$ (adjusted for multiple comparisons using the Benjamini Hochberg correction) were considered to be significantly enriched within that corresponding module. Modules were named using their corresponding number as well as the top most significantly enriched GO category.

\section{Module cell-type enrichment}

Genes in each module were evaluated for cell-type enrichment from a previous study using a mineable set of genes obtained from RNA-seq of sorted cells from the adult mouse cerebral cortex (Zhang et al. 2014). The cell-type assignment for each gene was determined by identifying the max fragments per kilobase of transcript per million mapped reads (FPKM) for each gene in one of 
the seven cell populations included in the RNA-seq data set. Each module was evaluated for enrichment using hypergeometric tests to examine the overlap of module genes for genes in each cell type. The list of 12,978 genes downloaded from brainrnaseq.org was used as the background set, and module genes that had at least one cell-type assignment were used. A Benjamini Hochberg correction for multiple comparisons was performed using the p.adjust() function in $\mathrm{R}$ to obtain adjusted $P$-values of significant overlap from these tests.

\section{Identification of module-trait relationships}

Pairwise correlation analysis was used to test the relationships between module expression and cognitive measures. First, the standardized average expression for each module was calculated across all 156 strain/sex/age/mutation carrier group measures (setting average $=0$, standard deviation $=1$ ). To test the relationship of modules to cognition, we performed Spearman correlation analysis to identify the specific molecular networks that significantly related to individual differences in cognitive outcomes. Using the previously reported (Neuner et al. 2019a) cognitive measures for each corresponding strain/sex/age/mutation carrier grouping, Spearman correlations for module average expression to measures for working memory (assessed using the Y-maze task), short-term memory, and long-term memory (assessed using CFC) were calculated. We chose the Spearman correlation approach over Pearson correlations because when we tested for normality of cognitive outcomes and module expression across the population, while cognitive outcomes were normally distributed (Supplemental Fig. S4), module expression was not (Supplemental Table S9). Modules that were significantly correlated (Benjamini Hochberg adjusted $P<0.05)$ with at least one of these three traits were selected for inclusion of downstream Bayesian direct acyclic graph analysis.

\section{ANOVA analysis of module expression on binary-coded traits}

Possible sources of variation in module expression were evaluated using a Type 2 ANOVA as a function of mutation carrier + age + sex (where mutation carrier denotes the presence/absence of the 5XFAD transgene) (Supplemental Fig. S1; Supplemental Table S3). All $P$-values were adjusted using the Benjamini Hochberg correction for multiple comparisons.

\section{Calculation of variance explained}

Effect size was calculated by generating a linear model using the $\mathrm{lm}$ () function in $\mathrm{R}$, where formula $=$ dependent $\sim$ independent. In this formula, dependent variables corresponded to the cognitive measure for working memory (percent spontaneous alternations), short-term memory (CFA slope), or long-term memory (percent freezing during CFC testing, CFM), and the independent variable was the standardized average expression for a specific module. A Type 2 ANOVA was performed, and the variance explained was obtained by taking the sum of squares for module expression and dividing by the total sum of squares for each model, which also corresponded to the multiple $R^{2}$ for each linear model. This analysis was performed for each module that was significantly correlated to working, short-term, and long-term memory measures. To determine the variance explained in AD-BXD individuals when amount of amyloid was added into the model, we first used a Type 3 ANOVA to test for significant interactions between module expression and amyloid amount. After identifying only one significant module*amyloid interaction (module $4^{*}$ amyloid for Y-maze $\%$ SA), we continued with an additive Type 2 ANOVA model. For this model, the same protocol was used as before in which variance explained was calculated using individual sum of squares for each independent variable divided by the total sum of squares (including residual sum of squares). Likelihood-ratio (LRT) tests were performed to determine if the addition of cortical A $\beta 1-42$ into the linear model significantly improved the fit. In this calculation, model 1: cognitive outcome $\sim$ module expression was tested against model 2: cognitive outcome $\sim$ module expression + cortical
A 1-42 ELISA to obtain a $P$-value which reflected if the two models were significantly different from each other. LRT tests with $P<0.05$ were interpreted to have a better fit with the addition of cortical A $1-42$ than with module expression alone. Results of ANOVA analyses and LRT tests are depicted in Supplemental Table S8.

\section{Module-trait network}

The module-trait network was constructed using the predefined relationships of modules that significantly correlated with at least one cognitive outcome, as well as genetic information provided by quantitative trait locus (QTL) mapping of module eigengenes. This module-trait network of mixed node times illustrated conditional dependencies among all variables (module expression, cognitive measures), and utilized genomic priors (genetic regions associated with module eigengenes) to help determine the orientation of edges in the resulting network (Tasaki et al. 2015; Mostafavi et al. 2018).

\section{Module QTL mapping}

Quantitative trait locus (QTL) mapping was performed using the eigengene for each module significantly correlated with cognitive outcomes. Genotypes for the BXD strains used in this study were obtained from GeneNetwork.org (Mulligan et al. 2017), and module eigengenes (ME) were generated by calculating the first principal component (PC1) of module expression. The BXD genotypes and module eigengenes were used for downstream QTL mapping in r/qtl (Broman et al. 2003). ANOVA analyses of module expression (Supplemental Fig. S1) were used to establish age sex, and/or genotype as additive covariates for each module. Covariates were used as additive, and 1000 permutations were used to determine statistical significance. Results of all QTL mapping is depicted in Supplemental Table S4.

\section{Ingenuity pathway analysis}

Ingenuity Pathway Analysis (IPA) was performed to complement the initial GO enrichment for gene coexpression modules. Specifically, the top enriched canonical pathways (by enrichment $P$-value) in the IPA Canonical Pathway Analysis report were analyzed to gain a more specific understanding of enriched pathways in modules.

\section{Enzyme-linked immunosorbent assay (ELISA)}

Data for beta-amyloid 1-42 (Aß1-42) quantification was used from ELISA arrays performed and described in our previously published study (Neuner et al. 2019a). Briefly, A $\beta 1-42$ levels were quantified from sections of temporal cortex [ 6 mo $n=72$ mice ( 46 female/46 male) across $22 \mathrm{AD}-\mathrm{BXD}$ strains, 14 mo $n=82$ mice ( 43 female/33 male) across 21 AD-BXD strains]. Tissue was homogenized in $1 \times$ PBS $+1 \%$ Triton X-100 using the TissueLyser II system (QIAGEN) and sonicated $2 \times 10 \mathrm{sec}$ on low power. Protein concentration was determined using a NanoDrop 2000 UV-Vis Spectrophotometer (ThermoScientific). Brain homogenates $(10 \mathrm{mg} / \mathrm{mL})$ were extracted in a final concentration of $5 \mathrm{M} \mathrm{GuHCl}$ overnight at $4^{\circ} \mathrm{C}$. Samples were then diluted appropriately and run in duplicate on A $31-42$-specific sandwich colorimetric ELISAs according to the manufacturer's protocol (Cat\# 298-92401, Wako Chemicals). Optical densities at $450 \mathrm{~nm}$ were read on a Biotek plate reader (BioTek) and $A \beta 1-42$ concentration was determined by comparison with A $1-42$ standard curves. Only readings in the linear range of the standard curve were included in analysis. Duplicates were averaged to determine concentration of A $\beta 1-42$ in each sample. Finally, A $\beta 1-42$ concentrations were normalized to total protein concentration and are reported as nanograms of $A \beta 1-42$ per milligrams of total protein.

\section{Mouse-to-human module comparisons}

Gene coexpression modules formed by using SpeakEasy clustering on bulk RNA-seq from human dorsolateral prefrontal cortex (DL-PFC) tissue were obtained from a previously published 
Religious Orders Study and Memory and Aging Project (ROSMAP) data set (Mostafavi et al. 2018), as well as coexpression modules from multiple human brain regions from the Accelerating Medicine Partnership- Alzheimer's Disease (AMP-AD) consortium (Wan et al. 2020). In the Mostafavi study, there were 47 modules formed with at least 20 gene members, and modules were related to human cognitive outcomes using a correlation approach similar to what was performed in this current study. In the AMP-AD study, there were 30 primary modules generated across seven brain regions and three studies compiled from a meta-analysis of human aged and AD brains. Human genes from these modules were converted to orthologous mouse genes using the biomaRt() package in R. Hypergeometric tests were used between each human and mouse module to examine the degree of conservation between the two species. The background gene list used was the total number of unique genes that could be found in any human or mouse module. The Benjamini-Hochberg correction for multiple comparisons was performed to obtain adjusted $P$-value using the p.adjust() function in R. All human and mouse modules that passed the preliminary filtering step (mouse modules $>29$ genes, Mostafavi human modules $>20$ genes, all AMP-AD modules) were examined for this analysis.

\section{Statistical analysis and software}

$\mathrm{R}$ software version 3.6.3 was used for data analysis. Code for SpeakEasy clustering was obtained from an online repository (https://www.cs.rpi.edu/ szymansk/SpeakEasy/index.html)

(Gaiteri et al. 2015), and run in Matlab version 2018a. Statistical tests included ANOVA, hypergeometric tests for overlap, Spearman correlations, and Benjamini Hochberg corrections for multiple testing. All scripts used for these analyses have been posted for open access to GitHub (Heuer_2020) and data files on Synapse.org (10.7303/syn17016211). Code for Bayesian modeling was obtained from an online repository at Synapse.org (10.7303/ syn2910187) (Tasaki et al. 2015).

\section{Acknowledgments}

This study is part of the National Institute on Aging (NIA) Resilience-AD program and is supported through the NIA grant award R01AG057914 to C.C.K., and R01AG057911 and 1R01AG061798 to C.G. This work was also supported by the NIA (AG054180 to C.C.K. and F31AG050357 to S.M.N.), the NIH (T32AG062409 to S.E.H.), and Bright Focus Foundation (A2016397S to C.C.K.). Additional support was provided by the Evnin family and the Burroughs Wellcome training fellowship.

Author contributions: S.E.H. and C.C.K. conceived of experiments. S.E.H., S.M.N., C.G., and C.C.K. designed and implemented experiments and analysis. S.E.H., N.H., C.C.K., and V.M.P. interpreted, designed, and implemented statistical analysis. S.M.N. conducted behavioral experiments, ELISA assays, and extracted RNA for sequencing. C.C.K., C.G., V.M.P., K.M.S.O., R.W.W., S.M.N, and N.H. assisted S.E.H. in designing experiments and interpretation of results, as well as preparation of manuscript. All authors reviewed and approved of the final manuscript.

\section{REFERENCES}

Allnutt MA, Johnson K, Bennett DA, Connor SM, Troncoso JC, Pletnikova O, Albert MS, Resnick SM, Scholz SW, De Jager PL, et al. 2020. Human herpesvirus 6 detection in Alzheimer's disease cases and controls across multiple cohorts. Neuron 105: 1027-1035.e1022. doi:10 .1016/j.neuron.2019.12.031

Anderson MA, Burda JE, Ren Y, Ao Y, O'Shea TM, Kawaguchi R, Coppola G, Khakh BS, Deming TJ, Sofroniew MV. 2016. Astrocyte scar formation aids central nervous system axon regeneration. Nature 532: 195-200. doi:10.1038/nature17623

Arboleda-Velasquez JF, Lopera F, O'Hare M, Delgado-Tirado S, Marino C, Chmielewska N, Saez-Torres KL, Amarnani D, Schultz AP, Sperling RA, et al. 2019. Resistance to autosomal dominant Alzheimer's disease in an APOE3 Christchurch homozygote: a case report. Nat Med 25: 16801683. doi:10.1038/s41591-019-0611-3
Arenaza-Urquijo EM, Vemuri P. 2018. Resistance vs resilience to Alzheimer disease: clarifying terminology for preclinical studies. Neurology 90: 695703. doi:10.1212/wnl.0000000000005303

Ben Menachem-Zidon O, Avital A, Ben-Menahem Y, Goshen I, Kreisel T, Shmueli EM, Segal M, Ben Hur T, \& Yirmiya R. 2011. Astrocytes support hippocampal-dependent memory and long-term potentiation via interleukin-1 signaling. Brain Behav Immun 25: 1008-1016. doi:10 .1016/j.bbi.2010.11.007

Bennett DA, Schneider JA, Arvanitakis Z, Wilson RS. 2012. Overview and findings from the religious orders study. Curr Alzheimer Res 9: 628-645. doi: $10.2174 / 156720512801322573$

Bennett ML, Bennett FC, Liddelow SA, Ajami B, Zamanian JL, Fernhoff NB, Mulinyawe SB, Bohlen CJ, Adil A, Tucker A, et al. 2016. New tools for studying microglia in the mouse and human CNS. Proc Natl Acad Sci 113: E1738-E1746. doi:10.1073/pnas.1525528113

Bertram L, McQueen MB, Mullin K, Blacker D, Tanzi RE. 2007. Systematic meta-analyses of Alzheimer disease genetic association studies: the AlzGene database. Nat Genet 39: 17-23. doi:10.1038/ng1934

Boros BD, Greathouse KM, Gentry EG, Curtis KA, Birchall EL, Gearing M, Herskowitz JH. 2017. Dendritic spines provide cognitive resilience against Alzheimer's disease. Ann Neurol 82: 602-614. doi:10.1002/ana .25049

Boros BD, Greathouse KM, Gearing M, Herskowitz JH. 2019. Dendritic spine remodeling accompanies Alzheimer's disease pathology and genetic susceptibility in cognitively normal aging. Neurobiol Aging 73: 92-103. doi:10.1016/j.neurobiolaging.2018.09.003

Broman KW, Wu H, Sen S, Churchill GA. 2003. R/qtl: qTL mapping in experimental crosses. Bioinformatics 19: 889-890. doi:10.1093/ bioinformatics/btg 112

Budson AE, Solomon PR. 2012. New diagnostic criteria for Alzheimer's disease and mild cognitive impairment for the practical neurologist. Pract Neurol 12: 88-96. doi:10.1136/practneurol-2011-000145

Chen C, Kim JJ, Thompson RF, Tonegawa S. 1996. Hippocampal lesions impair contextual fear conditioning in two strains of mice. Behav Neurosci 110: 1177-1180. doi:10.1037//0735-7044.110.5.1177

Crick FH. 1958. On protein synthesis. Symp Soc Exp Biol 12: 138-163.

Crystal H, Dickson D, Fuld P, Masur D, Scott R, Mehler M, Masdeu J, Kawas C, Aronson M, Wolfson L. 1988. Clinico-pathologic studies in dementia: nondemented subjects with pathologically confirmed Alzheimer's disease. Neurology 38: 1682-1687. doi:10.1212/wnl.38.11 .1682

Cummings BJ, Su JH, Cotman CW. 1993. Neuritic involvement within bFGF immunopositive plaques of Alzheimer's disease. Exp Neurol 124: 315325. doi:10.1006/exnr.1993.1202

de Chaves EP, Narayanaswami V. 2008. Apolipoprotein E and cholesterol in aging and disease in the brain. Future Lipidol 3: 505-530. doi:10.2217/ 17460875.3.5.505

De Jager PL, Ma Y, McCabe C, Xu J, Vardarajan BN, Felsky D, Klein HU, White CC, Peters MA, Lodgson B, et al. 2018. A multi-omic atlas of the human frontal cortex for aging and Alzheimer's disease research. Sci Data 5: 180142 . doi:10.1038/sdata.2018.142

Drayman N, Patel P, Vistain L, Tay S. 2019. HSV-1 single-cell analysis reveals the activation of anti-viral and developmental programs in distinct sub-populations. Elife 8: e46339. doi:10.7554/eLife.46339

Drummond E, Wisniewski T. 2017. Alzheimer's disease: experimental models and reality. Acta Neuropathol 133: 155-175. doi:10.1007/ s00401-016-1662-x

Dunn AR, O'Connell KMS, Kaczorowski CC. 2019. Gene-by-environment interactions in Alzheimer's disease and Parkinson's disease. Neurosci Biobehav Rev 103: 73-80. doi:10.1016/j.neubiorev.2019.06.018

Elmaleh DR, Farlow MR, Conti PS, Tompkins RG, Kundakovic L, Tanzi RE. 2019. Developing effective Alzheimer's disease therapies: clinical experience and future directions. J Alzheimers Dis 71: 715-732. doi:10 $.3233 /$ jad-190507

Fahmy GH, Moftah MZ. 2010. Fgf-2 in astroglial cells during vertebrate spinal cord recovery. Front Cell Neurosci 4: 129. doi:10.3389/fncel.2010 .00129

Farmer BC, Kluemper J, Johnson LA. 2019. Apolipoprotein E4 alters astrocyte fatty acid metabolism and lipid droplet formation. Cells 8: 182 . doi:10.3390/cells8020182

Fox NC, Warrington EK, Freeborough PA, Hartikainen P, Kennedy AM, Stevens JM, Rossor MN. 1996. Presymptomatic hippocampal atrophy in Alzheimer's disease. A longitudinal MRI study. Brain 119: 2001-2007. doi:10.1093/brain/119.6.2001

Franciosi S, Choi HB, Kim SU, McLarnon JG. 2005. IL-8 enhancement of amyloid-beta (Abeta 1-42)-induced expression and production of pro-inflammatory cytokines and COX-2 in cultured human microglia. $J$ Neuroimmunol 159: 66-74. doi:10.1016/j.jneuroim.2004.10.006

Frost EE, Nielsen JA, Le TQ, Armstrong RC. 2003. PDGF and FGF2 regulate oligodendrocyte progenitor responses to demyelination. J Neurobiol 54: 457-472. doi:10.1002/neu.10158 
Gaiteri C, Chen M, Szymanski B, Kuzmin K, Xie J, Lee C, Blanche T, Chaibub Neto E, Huang SC, Grabowski T, et al. 2015. Identifying robust communities and multi-community nodes by combining top-down and bottom-up approaches to clustering. Sci Rep 5: 16361. doi:10.1038/ srep16361

Gao LB, Yu XF, Chen Q, Zhou D. 2016. Alzheimer's Disease therapeutics: current and future therapies. Minerva Med 107: 108-113.

Genin E, Hannequin D, Wallon D, Sleegers K, Hiltunen M, Combarros O, Bullido MJ, Engelborghs S, De Deyn P, Berr C, et al. 2011. APOE and Alzheimer disease: a major gene with semi-dominant inheritance. Mol Psychiatry 16: 903-907. doi:10.1038/mp.2011.52

Gentry EG, Henderson BW, Arrant AE, Gearing M, Feng Y, Riddle NC, Herskowitz JH. 2016. Rho kinase inhibition as a therapeutic for progressive supranuclear palsy and corticobasal degeneration. J Neurosci 36: 1316-1323. doi:10.1523/jneurosci.2336-15.2016

Gomez-Pinilla F, Cummings BJ, Cotman CW. 1990. Induction of basic fibroblast growth factor in Alzheimer's disease pathology. Neuroreport 1: 211-214. doi:10.1097/00001756-199011000-00009

Graham WV, Bonito-Oliva A, Sakmar TP. 2017. Update on Alzheimer's Disease Therapy and Prevention Strategies. Anпu Rev Med 68: 413-430. doi:10.1146/annurev-med-042915-103753

Hardy J, Selkoe DJ. 2002. The amyloid hypothesis of Alzheimer's disease: progress and problems on the road to therapeutics. Science 297: 353356. doi: $10.1126 /$ science. 1072994

Henderson BW, Greathouse KM, Ramdas R, Walker CK, Rao TC, Bach SV, Curtis KA, Day JJ, Mattheyses AL, Herskowitz JH. 2019. Pharmacologic inhibition of LIMK1 provides dendritic spine resilience against beta-amyloid. Sci Signal 12: 587. doi:10.1126/scisignal.aaw9318

Heppner FL, Ransohoff RM, Becher B. 2015. Immune attack: the role of inflammation in Alzheimer disease. Nat Rev Neurosci 16: 358-372. doi:10 $.1038 / \mathrm{nrn} 3880$

Herskowitz JH, Feng Y, Mattheyses AL, Hales CM, Higginbotham LA, Duong DM, Montine TJ, Troncoso JC, Thambisetty M, Seyfried NT, et al. 2013. Pharmacologic inhibition of ROCK2 suppresses amyloid- $\beta$ production in an Alzheimer's disease mouse model. J Neurosci 33: 19086-19098. doi:10.1523/ineurosci.2508-13.2013

Hohman TJ, Tommet D, Marks S, Contreras J, Jones R, Mungas D. 2017. Evaluating Alzheimer's disease biomarkers as mediators of age-related cognitive decline. Neurobiol Aging 58: 120-128. doi:10.1016/j .neurobiolaging.2017.06.022

Holcomb L, Gordon MN, McGowan E, Yu X, Benkovic S, Jantzen P, Wright K, Saad I, Mueller R, Morgan D, et al. 1998. Accelerated Alzheimer-type phenotype in transgenic mice carrying both mutant amyloid precursor protein and presenilin 1 transgenes. Nat Med 4: 97100. doi:10.1038/nm0198-097

Hong S, Beja-Glasser VF, Nfonoyim BM, Frouin A, Li S, Ramakrishnan S, Merry KM, Shi Q, Rosenthal A, Barres BA, et al. 2016. Complement and microglia mediate early synapse loss in Alzheimer mouse models. Science 352: 712-716. doi:10.1126/science.aad 8373

Hsu HC, Lu L, Yi N, Van Zant G, Williams RW, Mountz JD. 2007. Quantitative trait locus (QTL) mapping in aging systems. Methods Mol Biol 371: 321-348. doi:10.1007/978-1-59745-361-5_23

Hyman B, Tanzi RE. 2019. Effects of species-specific genetics on Alzheimer's mouse models. Neuron 101: 351-352. doi:10.1016/j.neuron.2019.01 .021

Johnson WE, Li C, Rabinovic A. 2007. Adjusting batch effects in microarray expression data using empirical Bayes methods. Biostatistics 8: 118-127. doi:10.1093/biostatistics/kxj037

Katzman R, Terry R, DeTeresa R, Brown T, Davies P, Fuld P, Renbing X, Peck A. 1988. Clinical, pathological, and neurochemical changes in dementia: a subgroup with preserved mental status and numerous neocortical plaques. Ann Neurol 23: 138-144. doi:10.1002/ana .410230206

Kawai T, Akira S. 2006. Innate immune recognition of viral infection. Nat Immunol 7: 131-137. doi:10.1038/ni1303

Kawai T, Akira S. 2007. Antiviral signaling through pattern recognition receptors. J Biochem 141: 137-145. doi:10.1093/jb/mvm032

Keane TM, Goodstadt L, Danecek P, White MA, Wong K, Yalcin B, Heger A, Agam A, Slater G, Goodson M, et al. 2011. Mouse genomic variation and its effect on phenotypes and gene regulation. Nature 477: 289-294. doi:10.1038/nature10413

Kim JJ, Jung MW. 2006. Neural circuits and mechanisms involved in Pavlovian fear conditioning: a critical review. Neurosci Biobehav Rev 30: 188-202. doi:10.1016/j.neubiorev.2005.06.005

Kinney JW, Bemiller SM, Murtishaw AS, Leisgang AM, Salazar AM, Lamb BT. 2018. Inflammation as a central mechanism in Alzheimer's disease. Alzheimers Dement (N Y) 4: 575-590. doi:10.1016/j.trci.2018.06.014

Kiyota T, Ingraham KL, Jacobsen MT, Xiong H, Ikezu T. 2011. FGF2 gene transfer restores hippocampal functions in mouse models of Alzheimer's disease and has therapeutic implications for neurocognitive disorders. Proc Natl Acad Sci 108: E1339-E1348. doi:10.1073/pnas.1102349108
Kunkle BW, Grenier-Boley B, Sims R, Bis JC, Damotte V, Naj AC, Boland A, Vronskaya M, van der Lee SJ, Amlie-Wolf A, et al. 2019. Genetic meta-analysis of diagnosed Alzheimer's disease identifies new risk loci and implicates Abeta, tau, immunity and lipid processing. Nat Genet $\mathbf{5 1}$ 414-430. doi:10.1038/s41588-019-0358-2

Lambert JC, Ibrahim-Verbaas CA, Harold D, Naj AC, Sims R, Bellenguez C, DeStafano AL, Bis JC, Beecham GW, Grenier-Boley B, et al. 2013. Meta-analysis of 74,046 individuals identifies 11 new susceptibility loci for Alzheimer's disease. Nat Genet 45: 1452-1458. doi:10.1038/ng.2802

Leek JT, Johnson WE, Parker HS, Jaffe AE, Storey JD. 2012. The sva package for removing batch effects and other unwanted variation in high-throughput experiments. Bioinformatics 28: 882-883. doi:10 $.1093 /$ bioinformatics/bts034

Li G, Li J, Otsuka Y, Zhang S, Takahashi M, Yamada K. 2020. A BODIPY-based fluorogenic probe for specific imaging of lipid droplets. Materials (Basel) 13: 677. doi:10.3390/ma13030677

Liddelow SA, Guttenplan KA, Clarke LE, Bennett FC, Bohlen CJ, Schirmer L, Bennett ML, Münch AE, Chung WS, Peterson TC, et al. 2017. Neurotoxic reactive astrocytes are induced by activated microglia. Nature 541: $481-487$. doi: $10.1038 /$ nature21029

Lue LF, Rydel R, Brigham EF, Yang LB, Hampel H, Murphy GM, Brachova L, Yan SD, Walker DG, Shen Y, et al. 2001. Inflammatory repertoire of Alzheimer's disease and nondemented elderly microglia in vitro. Glia 35: 72-79. doi:10.1002/glia.1072

Magy L, Mertens C, Avellana-Adalid V, Keita M, Lachapelle F, Nait-Oumesmar B, Fontaine B, Baron-Van Evercooren A. 2003. Inducible expression of FGF2 by a rat oligodendrocyte precursor cell line promotes CNS myelination in vitro. Exp Neurol 184: 912-922. doi:10 .1016/j.expneurol.2003.08.019

Marschallinger J, Iram T, Zardeneta M, Lee SE, Lehallier B, Haney MS, Pluvinage JV, Mathur V, Hahn O, Morgens DW, et al. 2020. Lipid-droplet-accumulating microglia represent a dysfunctional and proinflammatory state in the aging brain. Nat Neurosci 23: 194-208. doi:10.1038/s41593-019-0566-1

Miller JA, Woltjer RL, Goodenbour JM, Horvath S, Geschwind DH. 2013. Genes and pathways underlying regional and cell type changes in Alzheimer's disease. Genome Med 5: 48. doi:10.1186/gm452

Minter MR, Moore Z, Zhang M, Brody KM, Jones NC, Shultz SR, Taylor JM, Crack PJ. 2016. Deletion of the type-1 interferon receptor in APPSWE/ PS1 $\triangle \mathrm{E} 9$ mice preserves cognitive function and alters glial phenotype. Acta Neuropathol Commun 4: 72. doi:10.1186/s40478-016-0341-4

Morris JC, Storandt M, McKeel DW Jr, Rubin EH, Price JL, Grant EA, Berg L. 1996. Cerebral amyloid deposition and diffuse plaques in "normal" aging: evidence for presymptomatic and very mild Alzheimer's disease. Neurology 46: 707-719. doi:10.1212/WNL.46.3.707

Mostafavi S, Gaiteri C, Sullivan SE, White CC, Tasaki S, Xu J, Taga M, Klein HU, Patrick E, Komashko V, et al. 2018. A molecular network of the aging human brain provides insights into the pathology and cognitive decline of Alzheimer's disease. Nat Neurosci 21: 811-819. doi:10.1038/ s41593-018-0154-9

Moynagh PN, Williams DC, O’Neill LA. 1994. Activation of NF-kappa B and induction of vascular cell adhesion molecule-1 and intracellular adhesion molecule-1 expression in human glial cells by IL-1 . Modulation by antioxidants. J Immunol 153: 2681-2690.

Mulligan MK, Mozhui K, Prins P, Williams RW. 2017. GeneNetwork: a toolbox for systems genetics. Methods Mol Biol 1488: 75-120. doi:10 .1007/978-1-4939-6427-7_4

Murphy MP, LeVine H III. 2010. Alzheimer's disease and the amyloid-beta peptide. J Alzheimers Dis 19: 311-323. doi:10.3233/jad-2010-1221

Murtie JC, Zhou YX, Le TQ, Vana AC, Armstrong RC. 2005. PDGF and FGF2 pathways regulate distinct oligodendrocyte lineage responses in experimental demyelination with spontaneous remyelination. Neurobiol Dis 19: 171-182. doi:10.1016/j.nbd.2004.12.006

Narayanan M, Huynh JL, Wang K, Yang X, Yoo S, McElwee J, Zhang B, Zhang C, Lamb JR, Xie T, et al. 2014. Common dysregulation network in the human prefrontal cortex underlies two neurodegenerative diseases. Mol Syst Biol 10: 743. doi:10.15252/msb.20145304

Negash S, Xie S, Davatzikos C, Clark CM, Trojanowski JQ, Shaw LM, Wolk DA, Arnold SE. 2013. Cognitive and functional resilience despite molecular evidence of Alzheimer's disease pathology. Alzheimers Dement 9: e89-e95. doi:10.1016/j.jalz.2012.01.009

Neuner SM, Wilmott LA, Hope KA, Hoffmann B, Chong JA, Abramowitz J, Birnbaumer L, O'Connell KM, Tryba AK, Greene AS, et al. 2015. TRPC3 channels critically regulate hippocampal excitability and contextual fear memory. Behav Brain Res 281: 69-77. doi:10.1016/j.bbr.2014.12 .018

Neuner SM, Hohman TJ, Richholt R, Bennett DA, Schneider JA, De Jager PL, Huentelman MJ, O'Connell KMS, Kaczorowski CC. 2017. Systems genetics identifies modifiers of Alzheimer's disease risk and resilience. bioRxiv 225714. doi:10.1101/225714

Neuner SM, Heuer SE, Huentelman MJ, O'Connell KMS, Kaczorowski CC. 2019a. Harnessing genetic complexity to enhance translatability of 
Alzheimer's disease mouse models: a path toward precision medicine. Neuron 101: 399-411.e395. doi:10.1016/j.neuron.2018.11.040

Neuner SM, Heuer SE, Zhang J-G, Philip VM, Kaczorowski CC. 2019b. Identification of pre-symptomatic gene signatures that predict resilience to cognitive decline in the genetically diverse AD-BXD model. Front Genet 10: 35 . doi:10.3389/fgene.2019.00035

Noda M, Takii K, Parajuli B, Kawanokuchi J, Sonobe Y, Takeuchi H, Mizuno T, Suzumura A. 2014. FGF-2 released from degenerating neurons exerts microglial-induced neuroprotection via FGFR3-ERK signaling pathway. I Neuroinflammation 11: 76. doi:10.1186/1742-2094-11-76

Oakley H, Cole SL, Logan S, Maus E, Shao P, Craft J, Guillozet-Bongaarts A, Ohno M, Disterhoft J, Van Eldik L, et al. 2006. Intraneuronal beta-amyloid aggregates, neurodegeneration, and neuron loss in transgenic mice with five familial Alzheimer's disease mutations: potential factors in amyloid plaque formation. J Neurosci 26: 1012910140. doi:10.1523/jneurosci.1202-06.2006

Ohno M, Sametsky EA, Younkin LH, Oakley H, Younkin SG, Citron M, Vassar R, Disterhoft JF. 2004. BACE1 deficiency rescues memory deficits and cholinergic dysfunction in a mouse model of Alzheimer's disease. Neuron 41: 27-33. doi:10.1016/s0896-6273(03)00810-9

Onos KD, Sukoff Rizzo SJ, Howell GR, Sasner M. 2016. Toward more predictive genetic mouse models of Alzheimer's disease. Brain Res Bull 122: 1-11. doi:10.1016/j.brainresbull.2015.12.003

Paterson RW, Toombs J, Slattery CF, Schott JM, Zetterberg H. 2014. Biomarker modelling of early molecular changes in Alzheimer's disease. Mol Diagn Ther 18: 213-227. doi:10.1007/s40291-013-0069-9

Peirce JL, Lu L, Gu J, Silver LM, Williams RW. 2004. A new set of BXD recombinant inbred lines from advanced intercross populations in mice. BMC Genet 5: 7. doi:10.1186/1471-2156-5-7

Pfrieger FW. 2003. Cholesterol homeostasis and function in neurons of the central nervous system. Cell Mol Life Sci 60: 1158-1171. doi:10.1007/ s00018-003-3018-7

Poirier J, Baccichet A, Dea D, Gauthier S. 1993. Cholesterol synthesis and lipoprotein reuptake during synaptic remodelling in hippocampus in adult rats. Neuroscience 55: 81-90. doi:10.1016/0306-4522(93)90456-p

Raghupathy N, Choi K, Vincent MJ, Beane GL, Sheppard KS, Munger SC, Korstanje R, Pardo-Manual De Villena F, Churchill GA. 2018. Hierarchical analysis of RNA-seq reads improves the accuracy of allele-specific expression. Bioinformatics 34: 2177-2184. doi:10.1093/ bioinformatics/bty078

Rambold AS, Cohen S, Lippincott-Schwartz J. 2015. Fatty acid trafficking in starved cells: regulation by lipid droplet lipolysis, autophagy, and mitochondrial fusion dynamics. Dev Cell 32: 678-692. doi:10.1016/j devcel.2015.01.029

Readhead B, Haure-Mirande JV, Funk CC, Richards MA, Shannon P, Haroutunian V, Sano M, Liang WS, Beckmann ND, Price ND, et al. 2018. Multiscale analysis of independent Alzheimer's cohorts finds disruption of molecular, genetic, and clinical networks by human herpesvirus. Neuron 99: 64-82.e67. doi:10.1016/j.neuron.2018.05.023

Reuss B, von Bohlen und Halbach O. 2003. Fibroblast growth factors and their receptors in the central nervous system. Cell Tissue Res 313: 139_ 157. doi:10.1007/s00441-003-0756-7

Ryman DC, Acosta-Baena N, Aisen PS, Bird T, Danek A, Fox NC, Goate A, Frommelt P, Ghetti B, Langbaum JB, et al. 2014. Symptom onset in autosomal dominant Alzheimer disease: a systematic review and meta-analysis. Neurology 83: 253-260. doi:10.1212/wnl 0000000000000596

Satorra A, Saris WE. 1985. Power of the likelihood ratio test in covariance structure analysis. Psychometrika 50: 83-90. doi:10.1007/BF02294150

Selkoe DJ. 1991. The molecular pathology of Alzheimer's disease. Neuron 6: 487-498. doi:10.1016/0896-6273(91)90052-2

Selkoe DJ, Hardy J. 2016. The amyloid hypothesis of Alzheimer's disease at 25 years. EMBO Mol Med 8: 595-608. doi:10.15252/emmm.201606210
Shobab LA, Hsiung G-YR, Feldman HH. 2005. Cholesterol in Alzheimer's disease. Lancet Neurol 4: 841-852. doi:10.1016/S1474-4422(05)70248-9 Sosna J, Philipp S, Albay R III, Reyes-Ruiz JM, Baglietto-Vargas D, LaFerla FM, Glabe CG. 2018. Early long-term administration of the CSF1R inhibitor PLX3397 ablates microglia and reduces accumulation of intraneuronal amyloid, neuritic plaque deposition and pre-fibrillar oligomers in 5XFAD mouse model of Alzheimer's disease. Mol Neurodegener 13: 11. doi:10.1186/s13024-018-0244-x

Spangenberg E, Severson PL, Hohsfield LA, Crapser J, Zhang J, Burton EA, Zhang Y, Spevak W, Lin J, Phan NY, et al. 2019. Sustained microglial depletion with CSF1R inhibitor impairs parenchymal plaque development in an Alzheimer's disease model. Nat Commun 10: 3758. doi:10.1038/s41467-019-11674-z

Stopa EG, Gonzalez AM, Chorsky R, Corona RJ, Alvarez J, Bird ED, Baird A. 1990. Basic fibroblast growth factor in Alzheimer's disease. Biochem Biophys Res Commun 171: 690-696. doi:10.1016/0006-291x(90)91201-3

Tasaki S, Sauerwine B, Hoff B, Toyoshiba H, Gaiteri C, Chaibub Neto E. 2015. Bayesian network reconstruction using systems genetics data: comparison of MCMC methods. Genetics 199: 973-989. doi:10.1534/ genetics.114.172619

Taylor JM, Minter MR, Newman AG, Zhang M, Adlard PA, Crack PJ. 2014 Type-1 interferon signaling mediates neuro-inflammatory events in models of Alzheimer's disease. Neurobiol Aging 35: 1012-1023. doi:10 .1016/j.neurobiolaging.2013.10.089

Thiele C, Wunderling K, Leyendecker P. 2019. Multiplexed and single cell tracing of lipid metabolism. Nat Methods 16: 1123-1130. doi:10.1038/ s41592-019-0593-6

Wan Y-W, Al-Ouran R, Mangleburg CG, Perumal TM, Lee TV, Allison K, Swarup V, Funk CC, Gaiteri C, Allen M, et al. 2020. Meta-analysis of the Alzheimer's disease human brain transcriptome and functional dissection in mouse models. Cell Rep 32: 2. doi:10.1016/j.celrep.2020 .107908

Williams RW, Gu J, Qi S, Lu L. 2001. The genetic structure of recombinant inbred mice: high-resolution consensus maps for complex trait analysis. Genome Biol 2: Research0046. doi:10.1186/gb-2001-2-11-research0046

Williams RB, Chan EK, Cowley MJ, Little PF. 2007. The influence of genetic variation on gene expression. Genome Res 17: 1707-1716. doi:10.1101/ gr.6981507

Wood WG, Li L, Müller WE, Eckert GP. 2014. Cholesterol as a causative factor in Alzheimer's disease: a debatable hypothesis. J Neurochem 129: 559-572. doi:10.1111/jnc.12637

Woodbury ME, Ikezu T. 2014. Fibroblast growth factor-2 signaling in neurogenesis and neurodegeneration. J Neuroimmune Pharmacol 9: 92101. doi:10.1007/s11481-013-9501-5

Zamanian JL, Xu L, Foo LC, Nouri N, Zhou L, Giffard RG, Barres BA. 2012. Genomic analysis of reactive astrogliosis. J Neurosci 32: 6391-6410. doi:10.1523/JNEUROSCI.6221-11.2012

Zhang B, Gaiteri C, Bodea LG, Wang Z, McElwee J, Podtelezhnikov AA, Zhang C, Xie T, Tran L, Dobrin R, et al. 2013. Integrated systems approach identifies genetic nodes and networks in late-onset Alzheimer's disease. Cell 153: 707-720. doi:10.1016/j.cell.2013.03.030

Zhang Y, Chen K, Sloan SA, Bennett ML, Scholze AR, O'Keeffe S, Phatnani HP, Guarnieri P, Caneda C, Ruderisch N, et al. 2014. An RNA-sequencing transcriptome and splicing database of glia, neurons, and vascular cells of the cerebral cortex. J Neurosci 34: 11929-11947. doi:10.1523/jneurosci.1860-14.2014

Zhu Y, Chai YL, Hilal S, Ikram MK, Venketasubramanian N, Wong BS, Chen CP, Lai MK. 2017. Serum IL-8 is a marker of white-matter hyperintensities in patients with Alzheimer's disease. Alzheimers Dement (Amst) 7: 41-47. doi:10.1016/j.dadm.2017.01.001

Received April 17, 2020; accepted in revised form June 10, 2020. 


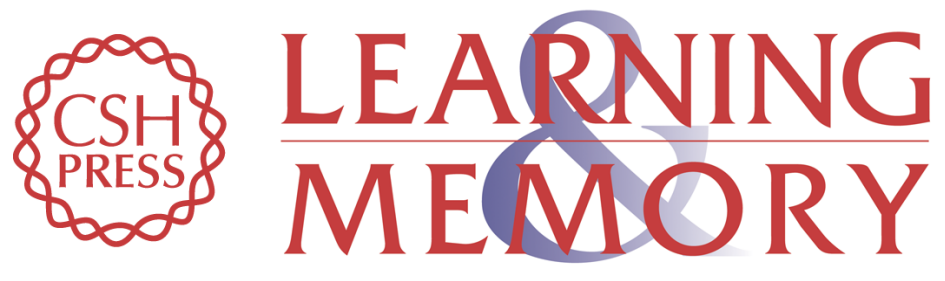

\section{Identifying the molecular systems that influence cognitive resilience to Alzheimer's disease in genetically diverse mice}

Sarah E. Heuer, Sarah M. Neuner, Niran Hadad, et al.

Learn. Mem. 2020, 27:

Access the most recent version at doi:10.1101//m.051839.120

Supplemental
Material http://learnmem.cshlp.org/content/suppl/2020/08/13/27.9.355.DC1

References This article cites 104 articles, 17 of which can be accessed free at: http://learnmem.cshlp.org/content/27/9/355.full.html\#ref-list-1

Creative This article, published in Learning \& Memory, is available under a Creative Commons Commons License (Attribution-NonCommercial 4.0 International), as described at License http://creativecommons.org/licenses/by-nc/4.0/.

Email Alerting Receive free email alerts when new articles cite this article - sign up in the box at the Service top right corner of the article or click here. 